\title{
Fundamental Limits to Near-Field Optical Response over Any Bandwidth
}

\author{
Hyungki Shim, ${ }^{1,2, *}$ Lingling Fan, ${ }^{1,3}$ Steven G. Johnson, ${ }^{4}$ and Owen D. Miller ${ }^{1, \dagger}$ \\ ${ }^{1}$ Department of Applied Physics and Energy Sciences Institute, Yale University, \\ New Haven, Connecticut 06511, USA \\ ${ }^{2}$ Department of Physics, Yale University, New Haven, Connecticut 06511, USA \\ ${ }^{3}$ School of Physics and National Laboratory of Solid State Microstructures, \\ Nanjing University, Nanjing 210093, China \\ ${ }^{4}$ Department of Mathematics, Massachusetts Institute of Technology, \\ Cambridge, Massachusetts 02139, USA
}

(Received 5 May 2018; revised manuscript received 11 December 2018; published 7 March 2019)

\begin{abstract}
We develop an analytical framework to derive upper bounds to light-matter interactions in the optical near field, where applications ranging from spontaneous-emission amplification to greater-than-blackbody heat transfer show transformative potential. Our framework connects the classic complex-analytic properties of causal fields with newly developed energy-conservation principles, resulting in a new class of power-bandwidth limits. These limits demonstrate the possibility of orders-of-magnitude enhancement in near-field optical response with the right combination of material and geometry. At specific frequency and bandwidth combinations, the bounds can be closely approached by canonical plasmonic geometries, with the opportunity for new designs to emerge away from those frequency ranges. Embedded in the bounds is a material "figure of merit," which determines the maximum response of any material (metal, dielectric, bulk, 2D, etc.), for any frequency and bandwidth. Our bounds on local density of states represent maximal spontaneous-emission enhancements, our bounds on cross density of states limit electromagneticfield correlations, and our bounds on radiative heat transfer (RHT) represent the first such analytical rule, revealing fundamental limits relative to the classical Stefan-Boltzmann law.
\end{abstract}

DOI: 10.1103/PhysRevX.9.011043

Subject Areas: Nanophysics, Optics, Photonics

\section{INTRODUCTION}

The electromagnetic near field comprises largeamplitude evanescent fields that can be harnessed to amplify spontaneous-emission rates [1-6], Casimir forces [7-11], Raman scattering [12-17], and greater-thanblackbody transfer of thermal energy [18-24]. Yet, little is known about maximal response-how large can such enhancements be? In this article, we derive fundamental limits for local density of states (LDOS), the prototypical near-field optical response, for any bandwidth of interest and for any material platform. We use these bounds to then derive fundamental limits for emerging quantities of interest-cross density of states (CDOS, a useful fieldcorrelation measure), and radiative heat transfer (RHT), where our bounds depend only on the temperatures, materials, and separation distance of the bodies involved.

\footnotetext{
*hyungki.shim@yale.edu

†owen.miller@yale.edu
}

Published by the American Physical Society under the terms of the Creative Commons Attribution 4.0 International license. Further distribution of this work must maintain attribution to the author(s) and the published article's title, journal citation, and DOI.
Conceptually, the bounds arise because LDOS and related near-field quantities are given by the real (or imaginary) parts of causal linear-response functions. We use the complex-analytic properties of such functions to transform bandwidth-averaged response to that at a single, complexvalued frequency, where we develop generalized energyconservation constraints, ultimately leading to bounds over arbitrary bandwidths. A distinctive feature of our arbitrarybandwidth approach is that it predicts a simple material figure of merit (FOM) that determines the maximum possible response of any material (metal, dielectric, 2D, $3 \mathrm{D}$, etc.), for any frequency and bandwidth. In the case of RHT, this FOM provides insight into which materials can facilitate optimal heat transfer for any temperature. There is significant ongoing debate about whether a plasmonic or an all-dielectric approach is better and in which scenarios 2D materials might be better than conventional bulk materials. Unlike all previous bounds and sum rules [24-43], the material figure of merit we derive here enables general quantitative answers to these questions. In a frequencybandwidth phase space, we map out which materials are optimal and where the critical thresholds, from dielectric to plasmonic and bulk to 2D, occur. The techniques developed herein for LDOS, CDOS, and radiative heat transfer should be extensible to other near-field effects ranging from 
engineered Lamb shifts [44,45] and Förster resonance energy transfer [46,47] to nonlinear (Raman) or fluctuation-induced (Casimir) phenomena.

Near-field electromagnetism, in which localized sources interact with scatterers separated by less than an optical wavelength, offers transformative potential for wideranging applications. Quantum emitters that only weakly couple to the radiation continuum can be dramatically amplified by near-field engineering: Optical antennas offer prospects for imaging single molecules $[13,14,48,49]$ or for designing nanoscale light-emitting diodes that are faster than lasers [50]. Nonlinear emitters such as Raman-active molecules [51,52] experience even more dramatic enhancements: Surface-enhanced Raman scattering (SERS) [12-14], for example, scales with the square of the spontaneous-emission enhancement rate. Thermal emission can be accessed and controlled for the productive transfer of heat energy, at rates many orders of magnitude beyond classical blackbodies [18-24]. The emission can be stimulated by the vacuum itself: The field of Casimir physics is probing a vast expanse of materials and structures to explore how vacuum forces can be controlled and manipulated [7-9,53-58].

For such a broad scope of applications, there is a fundamental theoretical question that remains unanswered: For a given bandwidth of interest, what is the maximum near-field response that is possible? Sum rules enable at least a partial answer. Relying on the analytic properties embedded within particular response functions-such as susceptibilities and cross sections-sum rules relate integrated response over all frequencies to behavior at a single frequency, and they have been derived in a variety of classical and quantum frameworks [25-33]. In the near field, there is a well-known sum rule for spontaneous emission [34] that suggests spontaneous-emission enhancements average out to zero over all frequencies. Yet, this sum rule neglects the longitudinal component of the Green's function, thus neglecting the near-to-far-field coupling that is crucial for spontaneous-emission engineering (and hence recovering a far-field refractive-index sum rule). Very recently, a near-field sum rule for electric LDOS was derived [35], which represents a specialized version of a sum rule that we derive in Eq. (4). However, sum rules make no predictions as to how a finite available bandwidth affects maximal response. The difference between infinite and finite bandwidth is stark for "dielectric" ( $\operatorname{Re} \epsilon>0$ ) materials because infinite-bandwidth sum rules include technologically irrelevant high-frequency contributions that dominate the integrated response. For example, when applied to Silicon, the sum rule for plane-wave scattering is dominated by contributions at energies on the order of $100 \mathrm{eV}$ [59], thus providing little insight into maximal response over typical bandwidths of interest (infrared, visible, etc.).

At the other extreme, single-frequency limits to power extinction and other physical observables have been discovered in both the near field $[24,36,37,43]$ and far field
$[38-42,60]$ based on energy-conservation principles, but they necessarily fail to account for the effects of nonzero bandwidth. (As an example, they predict infinite maximal response for any lossless material. Such a prediction is in fact correct-it is possible to make LDOS arbitrarily large [61]. But the bounds developed herein show that the average response over any nonzero bandwidth has a finite upper bound). Thus, previous approaches do not provide any meaningful metric for lossless dielectrics at optical frequencies, either for quantitative comparisons amongst each other or to plasmonic and other metallic systems.

The key idea of our work is that two seemingly independent ideas-causality for sum rules and energyconservation principles for single-frequency bounds - can be unified into a single framework that yield bounds for any bandwidth, as illustrated in Fig. 1. In this framework, single-frequency bounds and all-frequency sum rules emerge as asymptotic limits of a more general arbitrarybandwidth approach. Our bounds over arbitrary bandwidths, which we term "power-bandwidth limits," arise by connecting the properties that enable sum rules to those that enable energy-conservation principles. Sum rules for power quantities (such as optical cross section) require one to be able to compute the quantity by taking the imaginary (or real) part of some amplitude-for extinction, the optical theorem [33,62-64] guarantees such a form in terms of the scattering amplitude. The amplitude is a causal linearresponse function and thus analytic in the upper-half of the complex-frequency plane [65]. With suitable boundary conditions, a Hilbert transform (i.e., a Kramers-Kroniglike transform) then enables a sum rule, relating integrated response over all frequencies to that of a single frequency. Conversely, energy-conservation bounds-recognized primarily within the past decade $[24,36-43,60]$ —exploit the power-quantity-by-amplitude form in a different way. In writing a power quantity as the imaginary part of an amplitude, the amplitude itself is linear in the electromagnetic fields and/or currents (holding the incident field fixed). By contrast, many power quantities-absorption, scattering, radiation, etc.-are explicitly quadratic functionals of the fields and/or currents. If it can be shown that the linear quantity must be larger than the quadratic one, then an upper bound can be derived. Remarkably, it is the same optical theorem that provides such a constraint. Thus, we see that sum rules and single-frequency bounds both start with particular response functions that can be written as the imaginary part of an amplitude, but they diverge in their approaches thereafter. (For response functions that do not admit such expressions, the power-bandwidth limits established above can be applied by transformation to the real or imaginary part of a causal linear-response function.)

We connect the sum-rule and single-frequency approaches through the use of a "window function," an averaging function over a prescribed bandwidth. In general, such a function will have one (or multiple) poles in the 

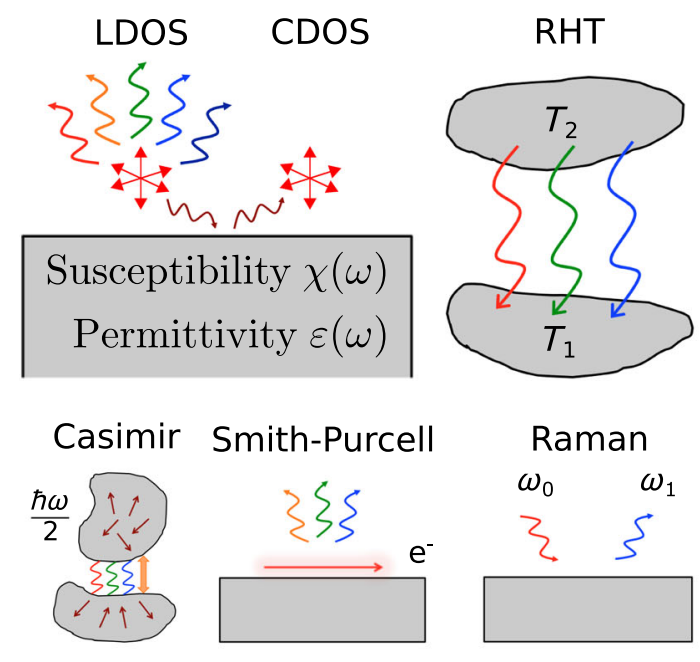

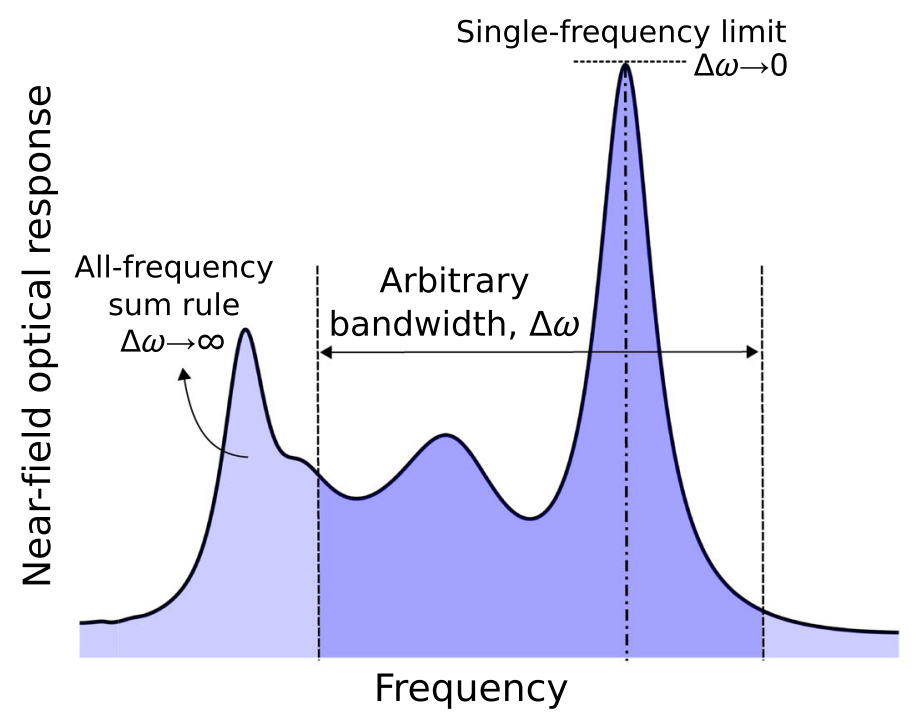

FIG. 1. Near-field optics exhibits phenomena ranging from spontaneous-emission enhancements through LDOS engineering, fieldcorrelation phenomena as measured by CDOS, RHT, Casimir effects, Smith-Purcell radiation, and Raman scattering. Our theoretical framework, connecting causality principles with energy-conservation constraints, yields bounds over any arbitrary bandwidth. In the limit of zero bandwidth, we obtain recently discovered single-frequency bounds [36,37]; in the infinite-bandwidth limit, we arrive at a sum rule for integrated all-frequency response.

upper-half plane (UHP), and thus the typical contourintegral analysis of a given power quantity requires the computation of residues not at a single real frequency (as in sum rules) but at a discrete set of complex frequencies. At this juncture, we identify the energy-conservation constraints at those complex frequencies, deriving bounds on how large they can be. This multistep procedure (fleshed out in detail below) thus provides perhaps the first approach to arbitrary-bandwidth bounds. For maximal clarity, we start with local density of states-the prototypical optical response-in Sec. II. We first derive near-field sum rules for LDOS (Sec. II A), showing that near-field response integrated over all frequencies must equal a new electrostatic constant, $\alpha_{\mathrm{LDOS}}$. Then, we use geometric perturbation theory to prove a monotonicity theorem that enables us to bound the electrostatic constant itself in terms of only the material permittivity and near-field separation distance (Sec. II B). We introduce the window function in Sec. II C, combining it with the complex-frequency energyconservation idea to develop arbitrary-bandwidth bounds and show how closely they can be approached for specific choices of frequency and bandwidth by canonical structures. Having established the theoretical bound framework, we derive general bounds for cross density of states (Sec. III) and near-field radiative heat transfer (Sec. IV). Emerging within all these bounds is a common material figure of merit, and in Sec. V, we discuss the physical intuition of the FOM and compare a wide variety of materials across frequency and bandwidth. Finally, in Sec. VI, we discuss extensions of our formulation to near-field phenomena such as Lamb shifts, Raman scattering, Casimir forces, and more.

\section{LOCAL DENSITY OF STATES}

In this section, we develop a theoretical framework for upper bounds to near-field optical-response functions. The prototypical near-field interaction is the alterationand potentially dramatic enhancement-of spontaneous emission from a two-level dipolar transition in a quantum emitter by an inhomogeneous environment. The power radiated by such an emitter, and thus the spontaneousemission rate enhancement, is proportional to the LDOS $[5,61,66-70]$. It has long been recognized that changing the electromagnetic environment of an emitter alters its spontaneous-emission rate [71-73]; applications where such enhancements play an important role include singlemolecule imaging [13,14], micro-LED design [74], and photovoltaics [75]. Mathematically, the spontaneousemission rate is determined by the imaginary part of the total Green's function [70,76,77]. To avoid unwieldy expressions and derivations, we use six-vector notation for fields and currents, treating the electric and magnetic fields, and electric and magnetic dipolar transitions, on equal footing. (We take the background to be vacuum throughout this paper and work in dimensionless units such that $\varepsilon_{0}=\mu_{0}=1$, with generalization to nonvacuum background in the Supplemental Material (SM) [78].) We denote the fields $\psi$, the polarization currents $\phi$, and dipolar sources $\xi$ :

$\psi=\left(\begin{array}{c}\mathbf{E} \\ \mathbf{H}\end{array}\right), \quad \phi=\left(\begin{array}{c}\mathbf{P} \\ \mathbf{M}\end{array}\right), \quad$ and $\quad \xi=\left(\begin{array}{l}\mathbf{p} \\ \mathbf{m}\end{array}\right)$.

Then, the spontaneous-emission rate of randomly oriented electric (p) and magnetic (m) dipoles at a point $\mathbf{x}_{0}$ is 

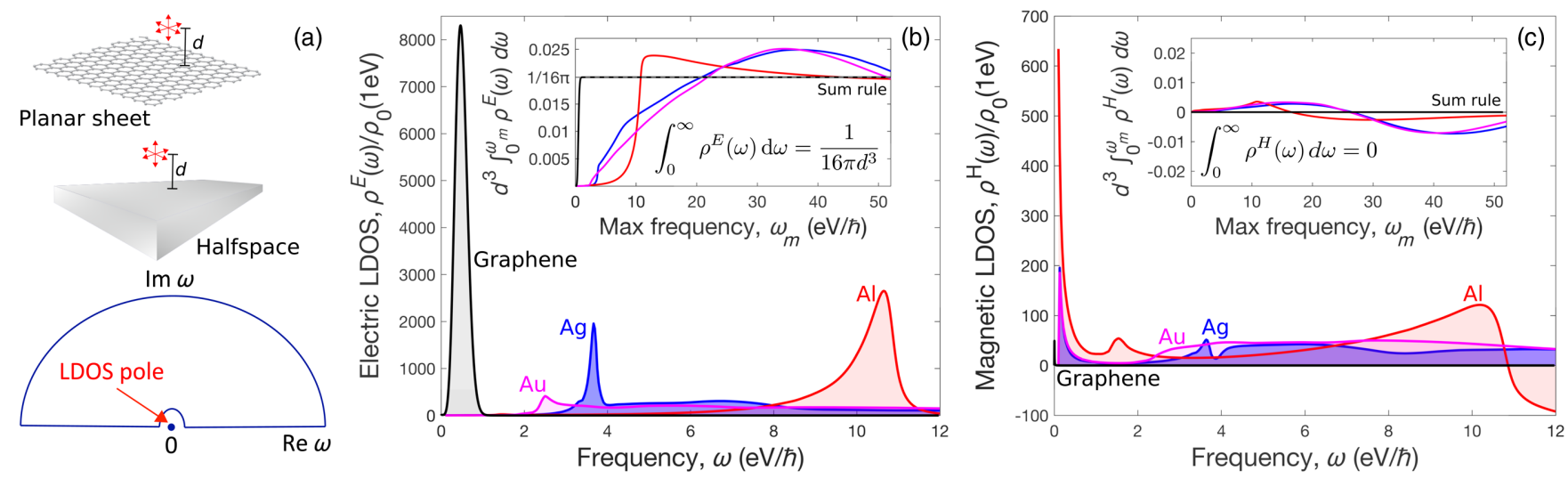

FIG. 2. (a) Contour of integration in the complex- $\omega$ plane used to obtain the sum rule for LDOS, which has a simple pole at the origin and is analytic everywhere on the upper half plane. For high-symmetry geometries such as a half-space or planar sheet, the sum-rule constant is known analytically. (b) Electric and (c) magnetic LDOS for Ag, Al, Au (half-spaces), and graphene (planar sheet). Although they all have resonant peaks at different frequencies with varying amplitudes and widths, their integrated response converges to the same constant, $1 / 16 \pi d^{3}$, as shown in the inset. The emitter-scatterer distance $d$ is set to $10 \mathrm{~nm}$, and the Fermi level for graphene is set to $0.6 \mathrm{eV}$.

modified relative to its free-space rate by the scattered-field contribution to the LDOS:

$$
\begin{aligned}
\rho\left(\mathbf{x}_{0}, \omega\right) & =\operatorname{Im} \sum_{j}\left[\frac{1}{\pi \omega}\left(\overline{\mathbf{p}}_{j} \cdot \mathbf{E}_{\mathrm{s}, j}\left(\mathbf{x}_{0}\right)+\overline{\mathbf{m}}_{j} \cdot \mathbf{H}_{\mathrm{s}, j}\left(\mathbf{x}_{0}\right)\right)\right] \\
& =\operatorname{Im} \underbrace{\sum_{j}\left[\frac{1}{\pi \omega} \xi_{j}^{\dagger}\left(\mathbf{x}_{0}\right) \psi_{\mathrm{s}, j}\left(\mathbf{x}_{0}\right)\right]}_{s(\omega)} \\
& =\operatorname{Im} \operatorname{Tr}\left[\frac{1}{\pi \omega} \boldsymbol{\Gamma}_{\mathrm{s}}\left(\mathbf{x}_{0}, \mathbf{x}_{0}, \omega\right)\right],
\end{aligned}
$$

where $\Gamma$ denotes the $6 \times 6$ dyadic Green's function, the "s" subscripts denote scattered-field contributions (thus, $\boldsymbol{\Gamma}_{s}$ is the total Green's function minus the free-space Green's function), and we use the convention [68] that LDOS is the sum of electric-dipole and magnetic-dipole contributions. It is important to subtract the free-space rate and consider only the scattered-field contribution, to ensure sufficiently fast decay at high frequency and thus convergence of integrals over frequency. The random dipole orientation (for dipoles of unit amplitude, i.e., $\xi^{\dagger} \xi=1$ ) is encoded in the summation over directions $j=$ $\{x, y, z\}$ and, ultimately, the trace of the Green's function. In Eq. (2), we denote a term $s(\omega)$ (suppressing the implicit position dependence), which we identify as a near-field scattering amplitude, as measured at the location of the emitter. It is this term that enables the sum rule and the power-bandwidth limits.

Maxwell's equations do not prevent us from taking the frequency to be complex. In the complex- $\omega$ plane, we can define the complex extension of the near-field scattering amplitude as

$$
s(\omega)=\sum_{j} \frac{1}{\pi \omega} \xi_{j}^{T}\left(\mathbf{x}_{0}\right) \psi_{\mathrm{s}, j}\left(\mathbf{x}_{0}\right), \quad \omega \in \mathbb{C},
$$

where we have made the typical assumption that the dipole amplitudes are real valued, such that $\xi^{\dagger}=\xi^{T}$. (For complex dipole amplitudes, a few additional steps in the derivations below are needed, but the results remain unchanged.) Since $\xi$ is constant (analytic everywhere), and the scattered field $\psi_{\mathrm{s}}$ is a causal linear-response function [65], the amplitude $s(\omega)$ is analytic in the upper half of the complex- $\omega$ plane. This is analogous to the classical result that quantities such as refractive index and far-field scattering amplitudes are analytic in the upper half plane (UHP) [65]. On the real line, $s(\omega)$ has a pole at the origin due to the singularity in the $1 / \pi \omega$ prefactor and the fact that $\xi^{T} \psi_{s}$ (usually) has a nonzero value in the zero-frequency limit.

\section{A. Sum rules}

Thus, a sum rule can be derived for $\rho\left(\mathbf{x}_{0}, \omega\right)$ through contour integration of the scattering amplitude $s(\omega)$ in the UHP. If we enclose the UHP in a typical contour that is semicircular going to infinity and follows the real line with a "bump" at the origin [see Fig. 2(a)], then analyticity ensures that the total integral is zero. For local, linear susceptibilities, $s(\omega)$ falls off sufficiently rapidly as $\omega \rightarrow \infty$ (because the free-space contribution was subtracted out), such that the real-line integral is well defined and the contour at infinity does not contribute (shown explicitly in the SM [78]). Thus, there are two contributions to the integral: the (principal value of the) integral over the real line and the residue of the simple pole at zero. Because negative-real-frequency fields are conjugates of their positive counterparts [79], $\rho(-\omega)=$ $\rho(\omega)$, such that the real-line integral can be reduced to only positive frequencies, after which a few algebraic steps 
(SM [78]) yield a general expression for the value of $\rho\left(\mathbf{x}_{0}, \omega\right)$ integrated over all frequencies:

$$
\int_{0}^{\infty} \rho(\omega) d \omega=\alpha_{\mathrm{LDOS}}
$$

where $\alpha_{\mathrm{LDOS}}=\frac{1}{2} \operatorname{Re}\left[\left.\operatorname{Tr} \boldsymbol{\Gamma}_{s}\left(\mathbf{x}_{0}, \mathbf{x}_{0}\right)\right|_{\omega=0}\right]$. The electrostatic constant $\alpha_{\text {LDOS }}$ measures the scattered field at the position of the dipole source and is shown in the SM [78] to always be positive. Equation (4) marks our first result: Over all frequencies, integrated LDOS must equal an electrostatic constant. [An electric-only specialization of Eq. (4) was very recently discovered [35], albeit without the analytical bounds to follow.] For materials with an electrical conductivity (e.g., metals), $\alpha_{\mathrm{LDOS}}$ is independent of the value of the conductivity, for any geometry. More generally, Eq. (4) applies to any material (whose susceptibility decays in the limit $\omega \rightarrow \infty$ ), including the wide array of newly emerging $2 \mathrm{D}$ materials.

Some care is required with Eq. (4) in singular situations. At high-symmetry points near high-symmetry scatterers, e.g., at the center of a hemispherical bowl, LDOS may appear to not decay at frequencies going to infinity because the optical rays reflect off the perfect spherical interface and constructively interfere at the origin. However, any random deviation from a hemisphere, no matter how small, would destroy such interference at high enough frequencies, restoring the natural, sufficiently rapid decay. Hence, the correct approach to regularizing such singularities is to compute the sum rule for a hemisphere with imperfections and then take the limit as the imperfections go to zero, such that Eq. (4) still applies. (Such a procedure is a geometrical analog of the limiting absorption principle [80], defining "lossless" materials in the limit as loss goes to zero from above.)

For any scatterer, the constant $\alpha_{\text {LDOS }}$ can be found from an electrostatic calculation. In high-symmetry geometries, the calculation may be analytically tractable. Consider a half-space of permittivity $\varepsilon$ and permeability $\mu$. (In this paper, we consider materials that have scalar material susceptibilities, with tensor-valued generalizations in the SM [78].) The value of $\alpha_{\text {LDOS }}$ at a separation $d$ can be computed via the image charge within the half-space, leading to an expression (derived in the SM [78]) for $\alpha_{\mathrm{LDOS}}$, and thus of the frequency-integrated LDOS, of

$$
\int_{0}^{\infty} \rho(\omega) d \omega=\frac{1}{16 \pi d^{3}}\left[\frac{\varepsilon(0)-1}{\varepsilon(0)+1}+\frac{\mu(0)-1}{\mu(0)+1}\right],
$$

where $\varepsilon(0)$ and $\mu(0)$ are the zero-frequency (electrostatic or magnetostatic) permittivities and permeabilities, respectively. For metals and any material with a conductivity, the permittivity and/or permeability diverges in the zerofrequency limit, such that the corresponding term in square brackets in Eq. (5) simplifies to 1. This result is also the case for any 2D conductive sheet, which at zero frequency represents the same perfect-conductor boundary condition as a conductive 3D half-space. In the SM [78], we also derive the simple $\alpha_{\mathrm{LDOS}}$ expression for conductive spheres.

An interesting feature of the LDOS sum rule is that it depends only on zero-frequency behavior, where electric and magnetic fields decouple. For the nonmagnetic materials that are ubiquitous at optical frequencies, this implies very different behavior for electric-dipole sources (i.e., electric-dipole transitions) as compared to magnetic-dipole sources (magnetic-dipole transitions). To illustrate the difference, one can separately define electric LDOS $\rho^{E}$ as the component arising from the electric sources only, and magnetic $\operatorname{LDOS} \rho^{H}$ as arising from the magnetic sources only:

$$
\begin{aligned}
\rho(\omega) & =\rho^{E}(\omega)+\rho^{M}(\omega) \\
& =\operatorname{Im}\left[\frac{1}{\pi \omega}\left(\operatorname{Tr} \boldsymbol{G}^{E E}+\operatorname{Tr} \boldsymbol{G}^{H H}\right)\right],
\end{aligned}
$$

where $\boldsymbol{G}^{E E}$ and $\boldsymbol{G}^{H H}$ are the electric and magnetic dyadic Green's functions, respectively. The two terms in the square brackets in Eq. (5) correspond to these individual LDOS constituents. For a nonmagnetic half-space (which we take as conductive just for simplicity), the electric and magnetic LDOS sum rules are

$$
\begin{aligned}
& \int_{0}^{\infty} \rho^{E}(\omega) \mathrm{d} \omega=\frac{1}{16 \pi d^{3}}, \\
& \int_{0}^{\infty} \rho^{H}(\omega) \mathrm{d} \omega=0 .
\end{aligned}
$$

The magnetic LDOS must average out to zero because, in the zero-frequency limit, a magnetic dipole does not interact with a nonmagnetic medium.

Figures 2(b) and 2(c) illustrate the generality of the electric and magnetic LDOS sum rules for bulk metals $(\mathrm{Ag}, \mathrm{Al}, \mathrm{Au})$ [81] as well as 2D materials such as graphene (material model from Refs. [82,83]). In our figures, we normalize electric and magnetic LDOS by the free-space electric (or magnetic) LDOS, $\rho_{0}=\omega^{2} /\left(2 \pi^{2} c^{3}\right)$ which is half that from Ref. [68] as they consider total free-space LDOS. Each of these materials supports surface plasmonpolaritons [84], which are excited by near-field sources. These materials exhibit very different resonant frequencies and line widths, as seen in Figs. 2(b) and 2(c). In terms of electric LDOS in Fig. 2(b), graphene exhibits a very large and narrow-band response at infrared frequencies, whereas metals exhibit varying levels of maximum response, with inversely proportional bandwidths, at visible or ultraviolet energies. In contrast to the large order-of-magnitude enhancements for electric LDOS, the magnetic LDOS in Fig. 2(c) shows only limited response-note the scale of the $y$ axis in Fig. 2(c) relative to Fig. 2(b). The modest, 
fluctuating magnetic LDOS arises because of the small electric field generated by the magnetic source or, equivalently, because the magnetic source cannot induce any magnetization in nonmagnetic media. Across the wide variations of response seen for both electric and magnetic LDOS, for systems with different materials and dimensionality, the all-frequency response must converge to the sum rules of Eqs. (4)-(6), as shown in the insets of Figs. 2(b) and $2(\mathrm{c})$.

\section{B. All-frequency bounds}

Equation (4) is an equality for any geometry. For structures without a high degree of symmetry, the electrostatic constant $\alpha_{\text {LDOS }}$ would typically require an electrostatic computation. In this section, we use perturbation theory to derive a "monotonicity theorem," showing that if one material body (with static permittivity and permeability greater than 1) encloses a second body of the same material, the electrostatic constant $\alpha_{\text {LDOS }}$ must be larger for the first than for the second. With this result, we can bound the allfrequency integrated response for any geometry in terms of the analytically known $\alpha_{\text {LDOS }}$ for high-symmetry enclosures, yielding general analytical bounds.

Any outward deformation can be broken down into a sequence of outward perturbations. Thus, if one can show that any outward perturbation of a geometry must increase some response function, a "mononicity theorem" has been proved, guaranteeing that such a function increases for any outward deformation. Such theorems are known for electrostatic polarizability under plane-wave excitations $[31,85,86]$. To understand how $\alpha_{\text {LDOS }}$ changes under geometrical perturbations, we use a variational-calculus approach applicable to any frequency and then isolate the electrostatic behavior. Within the variational-calculus approach, quantities incorporating the displacement fields $\mathbf{D}$ and $\mathbf{B}$ as well as the scalar permittivity and permeability will be necessary, for which we define the six-vector field $\Psi$ and tensor $\nu$ :

$$
\Psi=\left(\begin{array}{l}
\mathbf{D} \\
\mathbf{B}
\end{array}\right), \quad \nu=\left(\begin{array}{ll}
\varepsilon \mathcal{I} & \\
& \mu \mathcal{I}
\end{array}\right),
$$

where $\mathcal{I}$ is the $3 \times 3$ identity matrix (and as discussed above, generalizations to anisotropic materials are included in the SM [78]).

Consider a scattering FOM such as LDOS. Under geometrical perturbations, the variation in the FOM can be written as an overlap integral between two fields: (1) a "direct" field, which is the response of the unperturbed geometry to the original source (e.g., a nearby dipolar emitter), and (2) an "adjoint" field, which is the response of the same unperturbed geometry to a source whose phase, amplitude, and position depend on the precise FOM [87,88]. For any FOM, if we write the displacement of the boundary in the normal direction as $\Delta h_{n}(\mathbf{x})$, the variation in the FOM can generally be written as an overlap integral of the direct and adjoint fields over the geometrical boundary [88]:

$$
\begin{aligned}
\Delta \alpha_{\mathrm{LDOS}}= & 2 \operatorname{Re} \int \Delta h_{n}\left[\Delta \varepsilon \mathbf{E}_{\|} \cdot \mathbf{E}_{\|}^{(\text {adj })}-\Delta\left(\varepsilon^{-1}\right) \mathbf{D}_{\perp} \cdot \mathbf{D}_{\perp}^{(\text {adj })}\right. \\
& \left.+\Delta \mu \mathbf{H}_{\|} \cdot \mathbf{H}_{\|}^{(\text {adj })}-\Delta\left(\mu^{-1}\right) \mathbf{B}_{\perp} \cdot \mathbf{B}_{\perp}^{(\text {adj })}\right] \\
= & 2 \operatorname{Re} \int \Delta h_{n}\left[\psi_{\|}^{T} \Delta \nu \psi_{\|}^{(\text {adj })}+\Psi_{\perp}^{T} \nu_{1}^{-1} \Delta \nu \nu_{0}^{-1} \Psi_{\perp}^{(\text {adj })}\right],
\end{aligned}
$$

where $\Delta \varepsilon=\varepsilon_{1}-\varepsilon_{0}, \Delta\left(\varepsilon^{-1}\right)=\varepsilon_{1}^{-1}-\varepsilon_{0}^{-1}$ (similarly for $\mu$ ), $\Delta \nu=\nu_{1}-\nu_{0}$, and $\nu_{1}$ and $\nu_{0}$ represent the material properties of the scatterer and its surroundings, respectively, while the "adj" superscript denotes adjoint field solutions. Implied in the above integral over the boundary is multiplication with an area element $d A$ along the boundary, where $\psi_{\|}$and $\psi_{\perp}$ denote the field components parallel and perpendicular to the locally flat boundary, respectively. We have explicitly used the electrostatic constant $\alpha_{\text {LDOS }}$ for the figure of merit since it is that constant for which monotonicity will apply. For any figure of merit $f$, the adjoint fields are a solution with source currents given by the functional derivatives $\partial f / \partial \psi^{T}$. From Eq. (3) and the discussion following Eq. (4), we know that $\alpha_{\mathrm{LDOS}}$ is given by

$$
\alpha_{\mathrm{LDOS}}=\frac{1}{2} \operatorname{Re} \sum_{j} \xi_{j}^{T}\left(\mathbf{x}_{0}\right) \psi_{\mathrm{s}, j}\left(\mathbf{x}_{0}\right),
$$

which means that for any given dipole orientation $j$, the adjoint source field is given by $\partial f / \partial \psi_{j}^{T}=\frac{1}{2} \xi_{j}\left(\mathbf{x}_{0}\right)$. This shows a unique circumstance: The dipolar sources for the adjoint field are exactly half of $\xi_{j}\left(\mathbf{x}_{0}\right)$, which were the original LDOS dipolar excitations, such that $\psi^{(\mathrm{adj})}=\frac{1}{2} \psi$ and $\Psi^{(\text {adj })}=\frac{1}{2} \Psi$. Moreover, at zero frequency, without any material or radiative losses, the fields can be chosen to be real valued. Thus, for materials with positive static permittivities and permeabilities that are greater than those of their surroundings at zero frequency $\left(\nu_{0}, \nu_{1}\right.$, and $\Delta \nu$ positivedefinite), Eq. (8) can be written as the integral of a positive quantity,

$\Delta \alpha_{\mathrm{LDOS}}=\operatorname{Re} \int \Delta h_{n}\left[\psi^{T}(\Delta \nu) \psi+\Psi_{\perp}^{T}\left(\nu_{1}^{-1} \Delta \nu \nu_{0}^{-1}\right) \Psi_{\perp}\right]>0$.

Equation (10) ensures that $\Delta \alpha_{\mathrm{LDOS}}$ is positive for any outward deformation $\left(\Delta h_{n}>0\right.$ everywhere on the boundary). Regardless of the size and shape of a given scatterer $\Omega_{1}$ with constant $\alpha_{\text {LDOS }}^{(1)}$, we can always enclose it by another object $\Omega_{2}$, whose constant $\alpha_{\text {LDOS }}^{(2)}$ must be larger than $\alpha_{\text {LDOS }}^{(1)}$, proving our monotonicity theorem: 


$$
\alpha_{\mathrm{LDOS}}^{(2)}>\alpha_{\mathrm{LDOS}}^{(1)} \quad \text { for } \Omega_{2} \supset \Omega_{1}
$$

By connecting this monotonicity theorem to the sum rule in Eq. (4), one can see that the integrated LDOS near a scatterer must increase with the size or shape of that scatterer. Note that although our derivation does not strictly apply to the limiting case of a conductive material with $\Delta \nu \rightarrow \infty$ (because $\varepsilon \rightarrow \infty$ or $\mu \rightarrow \infty$ ) in the zero-frequency limit, it does apply for any arbitrarily large but finite material susceptibility, and in the SM [78], we provide an alternative proof that confirms the validity of Eq. (11) for conductive materials.

The use of Eq. (8) assumes a smooth perturbation of the boundary. In the case of a region with "kinks," or sharp corners, such a boundary represents the limit of smooth deformations. Since the fields are finite and the discontinuity is a region of zero measure, it would not contribute to first order [87], and monotonicity would hold. (It is not clear whether monotonicity would hold for fractal surfaces.) Although not covered explicitly by our derivation, monotonicity would also hold for a gradient-index (at zero frequency) medium, if the increase in index is non-negative everywhere (and positive over some region) across the medium.

A simple application of the monotonicity theorem is to enclose the scatterer within some larger half-space, which is possible as long as there is a separating plane between the emitter and the scatterer. The electrostatic constant of any half-space is given in Eq. (5), involving only the separation distance and the zero-frequency material parameters. Since the material factors $(\varepsilon-1) /(\varepsilon+1)$ and $(\mu-1) /(\mu+1)$ are bounded above by 1 (for static material constants larger than 1), we can replace them with 1 in the upper bounds. By the monotonicity theorem, the all-frequency integrated LDOS for any structure enclosed by a half-space (which is separated from the emitter by a minimum distance $d$ ) must obey the general bounds for any material:

$$
\int_{0}^{\infty} \rho(\omega) \mathrm{d} \omega=\int_{0}^{\infty}\left[\rho^{E}(\omega)+\rho^{H}(\omega)\right] \leq \frac{1}{8 \pi d^{3}},
$$

and, for nonmagnetic materials:

$$
\begin{aligned}
& \int_{0}^{\infty} \rho^{E}(\omega) \mathrm{d} \omega \leq \frac{1}{16 \pi d^{3}}, \\
& \int_{0}^{\infty} \rho^{H}(\omega) \mathrm{d} \omega=0 .
\end{aligned}
$$

If the scatterer in question can be more tightly enclosed by another shape, such as a sphere or two half-spaces, one can replace the rhs of Eqs. (12) and (13) with the respective electrostatic constants to obtain a tighter bound on $\alpha_{\text {LDOS }}$.

Figure 3 shows the electric LDOS for an emitter at the center of an aluminum double cone (similar to a bowtie antenna [89]), computed with an open-source software
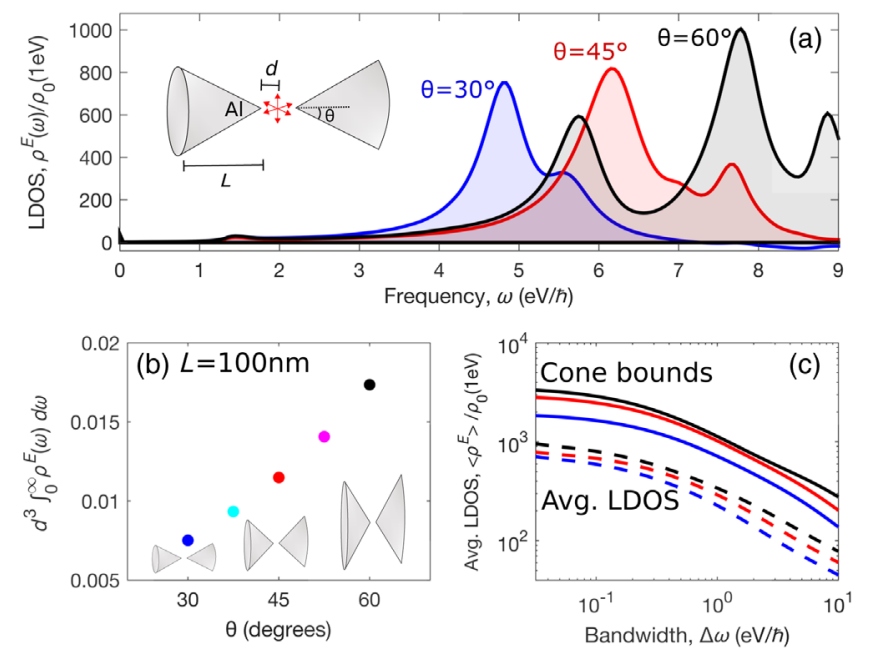

FIG. 3. (a) Electric LDOS for a double cone made of $\mathrm{Al}$ with $d=10 \mathrm{~nm}\left(L=15,25,35 \mathrm{~nm}\right.$ for $\theta=30^{\circ}, 45^{\circ}, 60^{\circ}$, respectively). (b) As the opening angle increases, the all-frequency integrated electric LDOS increases in confirmation of the monotonicity theorem. (c) Average electric LDOS centered at their peaks $\left(\omega_{0}=4.8,6.2,7.8 \mathrm{eV} / \hbar\right.$ for $\theta=30^{\circ}, 45^{\circ}, 60^{\circ}$, respectively) for each angle are shown along with their respective bounds. The double cones approach, within almost a factor of 2, their upper bounds, over a large range of bandwidths.

implementation [90,91] of the boundary element method (BEM) [92]. Figure 3(a) shows that for an emitter-antenna separation of $d=10 \mathrm{~nm}$ (a cone-cone separation of $20 \mathrm{~nm}$ ), thousandfold enhancements in electric LDOS are possible, at resonant frequencies determined by the geometry and the opening angle $\theta$. Enlarging the opening angle represents a way to increase the size of the scatterer, and Fig. 3(b) demonstrates a monotonic increase in integrated electric LDOS, as expected from the monotonicity theorem. In conjunction, the sum rule, Eq. (4), and the monotonicity theorem, Eq. (11), suggest a critical takeaway: Isolated sharp tips do not occupy enough of the near field to maximize electric LDOS; instead, large-area structured surfaces offer significantly greater potential.

Our identification of the causal linear-response function $s(\omega)$ defined in Eq. (2) as the underpinning of near-field sum rules ultimately yields a relation between all-frequency response and the single pole at the origin. Such relations form the crux of all sum rules [65], where the pole is almost always chosen at the origin or in the limit $\omega \rightarrow \infty$ (on the real line) because the response at those two particular frequencies can often be simplified: $\omega=0$ is the regime of electrostatics, while electromagnetism in the $\omega \rightarrow \infty$ limit is perturbative, as material susceptibilities converge to zero. From a theoretical viewpoint, of course, a pole can be introduced anywhere on the real line (not just $\omega \rightarrow 0, \infty$ ), or even anywhere in the UHP. Typically, however, one cannot make general statements about the response at arbitrary frequencies. In the next section, we show how to employ recently developed energy-conservation techniques to 

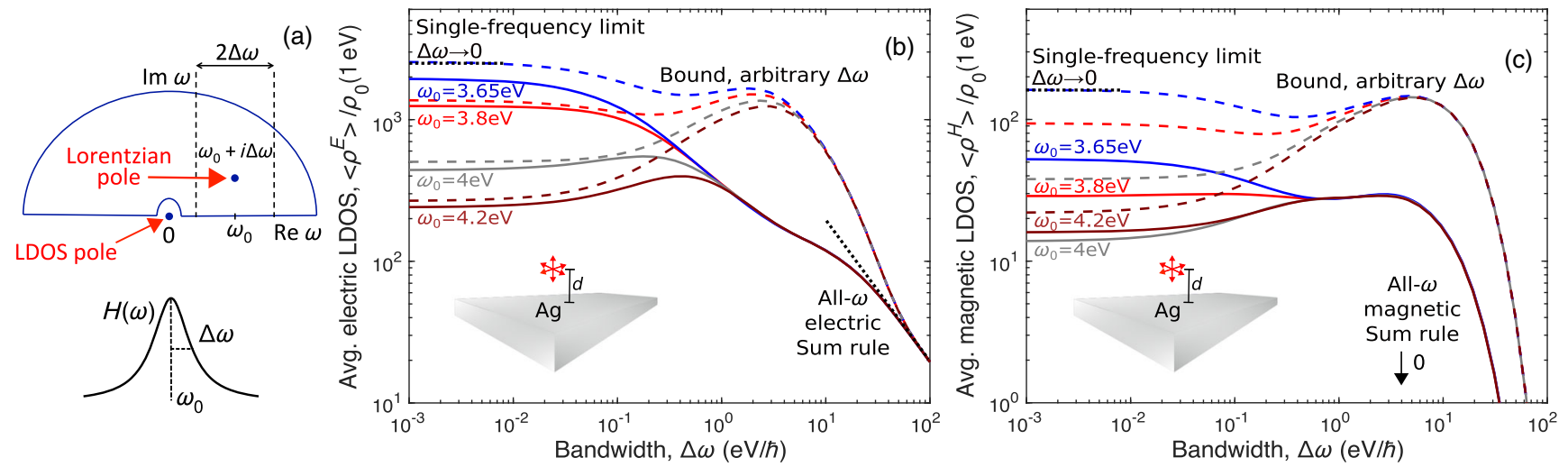

FIG. 4. (a) Contour of integration in the complex- $\omega$ plane used to obtain the average LDOS, which contains singularities at the origin ("LDOS pole") that is intrinsic to LDOS and at a complex frequency ("Lorentzian pole") determined by the parameters chosen for the Lorentzian window function $H_{\omega_{0}, \Delta \omega}$. Apart from these two poles, the product $\rho H_{\omega_{0}, \Delta \omega}$ is analytic everywhere on the upper half plane. (b) Average electric and (c) magnetic LDOS near a Ag half-space centered at different frequencies around its peak (3.65 eV), compared to their respective bounds. Taking the bandwidth to zero gives the single-frequency limit found in earlier works [36,37]. In the opposite limit of infinitely large bandwidth that includes the entire LDOS spectrum, our bounds reproduce the electric and magnetic sum rules in Sec. II A. The emitter-scatterer distance $d$ is set to $10 \mathrm{~nm}$.

derive general bounds at such frequencies, moving beyond the single-frequency or all-frequency dichotomy to a framework that works for any bandwidth.

\section{Power-bandwidth limits}

In this section, we introduce two ideas that transform the sum-rule approach of Sec. II A to an approach that bounds the response over any bandwidth: (1) We use the notion of a window function to connect the average response over some bandwidth to discrete frequencies in the upper half plane (at the window function's poles), and (2) we show how energy-conservation and passivity constraints can be applied at those complex frequencies, yielding analytical bounds on the bandwidth-averaged response.

Bandwidth plays a key role in any electromagnetic scattering problem, whether arising from the intrinsic line width of a source or as a primary technological constraint. For example, enhancements in LDOS over a broad spectrum could enable simultaneous imaging of multiple molecular species. Also, broadband LDOS enhancements provide a key criterion in designing optimal photovoltaic structures capable of maximal absorption enhancements [93].

There are many ways in which one might want to average the response over bandwidth (equal weighting, Lorentzian weighting, etc.), and one can accommodate almost any by prescribing a window function $H_{\omega_{0}, \Delta \omega}(\omega)$ that serves as a weighting function-it is concentrated around a center frequency $\omega_{0}$, defined by a frequency width $\Delta \omega$, and normalized $\left(\int_{-\infty}^{\infty} H_{\omega_{0}, \Delta \omega}(\omega) \mathrm{d} \omega=1\right)$. Then, the average LDOS over that range of frequencies, which we denote $\langle\rho\rangle$, can be defined by

$$
\langle\rho\rangle \equiv \int_{-\infty}^{\infty} \rho(\omega) H_{\omega_{0}, \Delta \omega}(\omega) \mathrm{d} \omega
$$

For the remainder of this section, we choose a Lorentzian function for $H_{\omega_{0}, \Delta \omega}$ :

$$
H_{\omega_{0}, \Delta \omega}(\omega)=\frac{\Delta \omega / \pi}{\left(\omega-\omega_{0}\right)^{2}+(\Delta \omega)^{2}},
$$

where $\Delta \omega$ is the half width at half maximum. We use a Lorentzian for simplicity: $H_{\omega_{0}, \Delta \omega}(\omega)$ extended into the UHP has only a single pole at $\omega=\omega_{0}+i \Delta \omega$. Other window functions can be used with the simple addition of extra (or higher-order [61]) poles. For example, one can approximate a rect function (where $H_{\omega_{0}, \Delta \omega}$ is constant over the bandwidth of interest and zero elsewhere) by the generalization $H_{\omega_{0}, \Delta \omega}(\omega)=\left[c(\Delta \omega)^{2 n-1}\right] /\left[\left(\omega-\omega_{0}\right)^{2 n}+(\Delta \omega)^{2 n}\right], \quad$ which has $n$ poles in the UHP. The bounds below at a single pole would then become a linear combination of the bounds at the new poles, with slightly modified numerical factors but the same physical ramifications for material and structural design.

Inserting the Lorentzian of Eq. (15) into the average LDOS [Eq. (14)] and writing the LDOS in terms of the near-field scattering amplitude, $\rho=\operatorname{Im} s(\omega)$, the product $\rho(\omega) H(\omega)$ in the averaging integrand is given by $\operatorname{Im}[s(\omega) H(\omega)]$. Outside of the lower-half plane, the function $s(\omega) H(\omega)$ has two simple poles: one at the origin, which was responsible for the sum rule from Sec. II A, and another at $\omega_{0}+i \Delta \omega$, from the Lorentzian, as shown in Fig. 4(a). One can integrate over the contour in Fig. 4(a) and use Cauchy's residue theorem to equate the allfrequency integral of Eq. (14) to the evaluation of two complex-frequency quantities:

$$
\langle\rho\rangle=\operatorname{Im} s\left(\omega_{0}+i \Delta \omega\right)+2 H_{\omega_{0}, \Delta \omega}(0) \alpha_{\mathrm{LDOS}},
$$


where $s\left(\omega_{0}+i \Delta \omega\right)$ is the near-field scattering amplitude evaluated at the single complex frequency $\omega_{0}+i \Delta \omega$, and $\alpha_{\text {LDOS }}$ is the electrostatic constant appearing in the sum rule defined in Eq. (4). In the limit of zero bandwidth, $H_{\omega_{0}, \Delta \omega}(0)$ equals zero, and Eq. (16) comprises only the first term, which represents single-frequency LDOS: $\rho=\operatorname{Im} s(\omega)$; conversely, as the bandwidth goes to infinity, $\operatorname{Im} s\left(\omega_{0}+\right.$ $i \Delta \omega$ ) decays rapidly (as we show below) and the second term converges to the sum rule [Eq. (4)]. Between these extremes, the two terms comprising $\langle\rho\rangle$ combine to represent a bandwidth-averaged response.

At the complex frequency $\omega_{0}+i \Delta \omega$, the positive imaginary part of the wave number $k$ means that the incident field emanating from the dipolar source is exponentially decaying, as can be understood from the outwardgoing wave $e^{i k r} / r \rightarrow e^{i \omega_{0} r / c} e^{-\Delta \omega r / c}$. The decaying source is mathematically equivalent to a scattering problem in which the frequency is real valued but material loss is increased in both the scatterer and the background [94]. Through either viewpoint, one can see that large broadbandwidth response is inherently more difficult to achieve than large single-frequency response due to an inherent bandwidth-induced dissipation.

By expressing the weighted integral of LDOS in terms of residues evaluated at single complex frequencies, Eq. (16) is now conceptually similar to single-frequency response functions at real frequencies for which we have developed an energy-conservation or passivity-based approach to upper bounds [36,37]. The key idea as applied here is that Eq. (16) is the imaginary part of a function that is linear in the scattering amplitude $s\left(\omega_{0}+i \Delta \omega\right)$, while representing the total (bandwidth-averaged) power lost by the dipolar source, to either far-field radiation or near-field absorption. By contrast, absorption itself is dissipation in the medium, computed with the imaginary part of the susceptibility and the field intensity $|\mathbf{E}|^{2}$, a function that is quadratic in the fields. Since absorption must be smaller than total LDOS (absorption + radiation), this implies that the quadratic functional must be smaller than the linear functional, a convex optimization constraint that necessarily bounds how large the fields and induced currents can be. We defer to Ref. [36] for a more detailed discussion of such single-frequency optimization, and we emphasize below the new developments in the case of a complex frequency.

To bound the first term, $s\left(\omega_{0}+i \Delta \omega\right)$, in Eq. (16) (the second term is the known electrostatic constant), it is helpful to rewrite the near-field scattering amplitude not in terms of the field at the source location but instead in terms of the fields within the scatterer, at the complex frequency $\omega=\omega_{0}+i \Delta \omega$. The amplitude can be written most succinctly in terms of the material susceptibility $\chi(\omega)$, which is the difference between the scatterer permittivity or permeability and that of the background: $\chi(\omega)=\nu(\omega)-\nu_{0}(\omega)=\left(\begin{array}{ll}(\varepsilon-1) \mathcal{I} & \\ & (\mu-1) \mathcal{I}\end{array}\right)$.

(In the SM [78], we treat the most general scenario in which the susceptibility is a $6 \times 6$ tensor that can be magnetic, anisotropic, nonreciprocal, and spatially inhomogeneous.) If we consider the LDOS for a single dipole orientation $j$ and momentarily drop the $j$ notation for simplicity, the scattering amplitude is, per Eq. (3), $s(\omega)=(1 / \pi \omega) \xi^{T}\left(\mathbf{x}_{0}\right) \psi_{s}\left(\mathbf{x}_{0}\right)$. The scattered field at the dipole location, $\psi_{s}\left(\mathbf{x}_{0}\right)$, is given by the convolution of the free-space Green's function $\boldsymbol{\Gamma}$ with the polarization currents $\phi=\chi \psi$ in the scatterer; then, reciprocity [95] can connect the free-space Green's function to the incident field from the dipole itself (a procedure we followed at real frequencies in Ref. [36]). After defining a modified incident field, $\tilde{\psi}_{\text {inc }}=$ $\left(\mathbf{E}_{\text {inc }}-\mathbf{H}_{\text {inc }}\right)^{T}$, the near-field scattering amplitude can be written as $s(\omega)=(1 / \pi \omega) \int_{V} \tilde{\psi}_{\text {ind }}^{T} \chi \psi$.

Finding a convex constraint that encodes energy conservation-requiring absorbed power (properly normalized) to be smaller than total power expended-requires some care at complex frequencies. One cannot analytically continue the absorbed and scattered powers into the UHP, as they are not analytic everywhere (originating from their quadratic field dependence). To recover the notion of an energy-conservation constraint, we start with passivity, which states that everywhere in the UHP, the product $\operatorname{Im}(\omega \chi)$ must be positive-definite [96]:

$$
\operatorname{Im}[\omega \chi(\omega)]>0 \quad \text { for } \operatorname{Im} \omega>0,
$$

where $\operatorname{Im}(\omega \chi)=\left[\omega \chi-(\omega \chi)^{\dagger}\right] / 2 i$, and Eq. (18) extends into the complex plane the notion that passivity implies positive(-definite) imaginary susceptibilities. From passivity, we define two positive functionals: an integral of the (positive) quantity $\operatorname{Im}\left[\psi^{\dagger}(\omega \chi) \psi\right]$ within the scatterer volume, and an integral of the (positive) quantity $\operatorname{Im}\left[\psi_{s}^{\dagger}(\omega \chi) \psi_{s}\right]$ outside the scatterer volume. Through repeated application of the divergence theorem and the complex-frequency Maxwell equations, we can define two new functionals, $\varphi_{A}(\omega)$ and $\varphi_{E}(\omega)$. At real frequencies, the functionals equal absorption and extinction, but we define them here in the UHP:

$$
\begin{aligned}
& \varphi_{A}(\omega)=\frac{1}{2} \operatorname{Im} \int_{V} \psi^{\dagger}(\omega \nu) \psi, \\
& \varphi_{E}(\omega)=\frac{1}{2} \operatorname{Im} \int_{V} \psi_{\text {inc }}^{\dagger}\left[\left(\omega \nu-\left(\omega \nu_{0}\right)^{\dagger}\right) \psi-\omega \nu_{0} \psi_{\text {inc }}\right] .
\end{aligned}
$$

In the SM [78], we show that these functionals indeed satisfy absorption and extinction-like constraints everywhere in the UHP: $\varphi_{A}(\omega)<\varphi_{E}(\omega)$ for $\operatorname{Im} \omega>0$. This constraint is precisely the type of convex constraint needed for a bound, providing a mechanism to derive one at complex 
frequencies. Given the expression above for $s(\omega)$, and the constraint $\varphi_{A}<\varphi_{E}$, we formulate the upper bound as the solution of a (convex) optimization problem at $\omega=$ $\omega_{0}+i \Delta \omega$ :

$$
\begin{aligned}
\underset{\psi(\mathbf{x}, \omega)}{\operatorname{maximize}} \operatorname{Im} s(\omega) & =\operatorname{Im}\left[\frac{1}{\pi \omega} \int_{V} \tilde{\psi}_{\text {inc }}^{T}(\omega) \chi(\omega) \psi(\omega)\right], \\
\text { subject to } \varphi_{A}(\omega) & \leq \varphi_{E}(\omega) .
\end{aligned}
$$

Equation (20) has a unique, globally optimal solution. The optimal field distribution $\psi(\omega)$ and scattering amplitude $s(\omega)$ can be found through variational calculus, following a similar procedure to that developed in Ref. [36] and detailed in the SM [78]. A crucial term that emerges is a material-dependent material figure of merit, $f(\omega)$. For bulk (non-2D), nonmagnetic materials with scalar electric susceptibilities $\chi(\omega)$, the material FOM is given by

$$
f\left(\omega=\omega_{0}+i \Delta \omega\right)=\frac{|\omega \chi|^{2}+|\omega \chi| \Delta \omega}{|\omega| \operatorname{Im}(\omega \varepsilon)} .
$$

The optimal field is proportional to this material function, as well as to the conjugate of the incident field. Now, reintroducing the average over dipole orientation $j$, the optimal field yields a frequency-averaged LDOS bound (SM [78]):

$$
\langle\rho\rangle \leq \frac{f(\omega)}{\pi|\omega|} \sum_{j} \int_{V} \psi_{\mathrm{inc}, j}^{\dagger}(\omega) \psi_{\mathrm{inc}, j}(\omega) \mathrm{d} V+2 H_{\omega_{0}, \Delta \omega}(0) \alpha_{\mathrm{LDOS}},
$$

where the complex frequency $\omega=\omega_{0}+i \Delta \omega$ encodes the center frequency and bandwidth of interest.

Equation (22) shows that a near-field LDOS, averaged in a half-width-at-half-max bandwidth $\Delta \omega$ around a center frequency $\omega_{0}$, is fundamentally limited by the field of a dipole in free space and by the frequency-dependent material composition of the scatterer(s). The volume integral of the incident field can be further simplified by enclosing the scatterer within some bounding shape of high symmetry over which the integral can be calculated analytically. A typical example is that of an emitter above a structured (or randomly textured [97,98]) surface, in which case the scatterer can be enclosed in a half-space that is a separation distance $d$ from the emitter. Near-field interactions $(|\omega| d / c \ll 1)$ are dominated by the rapidly decaying evanescent fields emanating from the sources, which implies that the overall shape of the scatterer, aside from its dimensionality, has little effect on the volume integral in Eq. (22). Enclosing any structure by a half-space and keeping only the dominant near-field term in the integral in Eq. (22), we obtain a simple, shape-independent, analytical expression. (All remaining terms, which are nondivergent and typically small, are included in the SM [78].) The bound scaling is very different when the incident field is generated by a magnetic dipole rather than an electric one, and thus we can separately derive for total LDOS $\rho$, electric LDOS $\rho^{E}$, and magnetic LDOS $\rho^{H}$, the general bandwidth-averaged bounds (SM [78]):

$$
\begin{gathered}
\frac{\langle\rho\rangle}{\rho_{0}(|\omega|)}, \frac{\left\langle\rho^{E}\right\rangle}{\rho_{0}(|\omega|)} \leq \frac{1}{8|k|^{3} d^{3}}\left(f(\omega) e^{-2 d \Delta \omega / c}+2 \frac{\Delta \omega}{|\omega|}\right), \\
\frac{\left\langle\rho^{H}\right\rangle}{\rho_{0}(|\omega|)} \leq \frac{1}{4|k| d} f(\omega) e^{-2 d \Delta \omega / c},
\end{gathered}
$$

where we have defined a complex-valued wave number $k=\omega / c$, the function $f(\omega)$ is the material FOM from Eq. (21), and for the second term on the first line, we have inserted the half-space bounds for $\alpha_{\text {LDOS }}$ from Eq. (13).

Equations. (22) and (23) are foundational results of our paper. No geometrical engineering of resonances or coupling can overcome their limits. For any structure and bandwidth, the bound of Eq. (23) depends only on the frequency range of interest, the material properties at those frequencies, and the emitter-scatterer separation.

Figure 4 compares the LDOS near a silver half-space to the bounds of Eq. (23). Center frequencies ranging from $\omega_{0}=3.65 \mathrm{eV} / \hbar$ to $\omega_{0}=4.2 \mathrm{eV} / \hbar$ (with corresponding wavelengths from $340 \mathrm{~nm}$ to $295 \mathrm{~nm}$ ) are considered near the surface-plasmon frequency of silver. One can show analytically that in the zero-bandwidth, near-field $(k d \ll 1)$ limit, the electric LDOS above a nonmagnetic, surfaceplasmon-resonant interface should approach the bound of Eq. (23), while the magnetic LDOS above the same interface should approach its respective bound within a factor of 2. (This can be shown starting from asymptotic expressions in Ref. [68].) Such close approaches in the zero-bandwidth limit are visible in both Figs. 4(b) and 4(c). In the large-bandwidth limit, the bounds converge to the sum rules of Sec. II A, ensuring that they are "tight" (i.e., there is no smaller upper bound) in that regime as well. To simplify the ultrahigh-bandwidth computations $(\Delta \omega \gtrsim 100 \mathrm{eV} / \hbar)$, we use a five-pole Drude-Lorentz multi-oscillator model for silver that closely approximates tabulated susceptibility data [81] (a comparison is included in the SM [78]). There is an interesting peak in the bound at moderate bandwidths that arises due to the large, lossy permittivity of silver at about $5 \mathrm{eV}$; standard quasistatic theory [99] would predict that a half-space is nonoptimal for such a bandwidth but that perhaps another structure is optimal. A key question prompted by these bounds is whether nonplanar, designed structures-or perhaps even randomly corrugated structures - can approach the bounds at frequencies away from the surface-plasmon resonance. In Fig. 3(c), it was observed that double cones approach within almost of factor of 2 of their upper bounds [using Eq. (22) for their specific geometry] over a large range of bandwidths, further suggesting that such structural design should be possible. 
The convergence of the electric-magnetic LDOS bounds to their respective sum rules, in the infinitebandwidth limit, can be verified directly from Eq. (23). The first term in the $\rho^{E}$ bound goes to zero as $\Delta \omega \rightarrow \infty$ due to the $e^{-2 d \Delta \omega / c}$ factor, in which case one can rewrite the bound as $\left\langle\rho^{E}\right\rangle \leq 1 /\left(8 \pi^{2} \Delta \omega d^{3}\right)=(2 / \pi \Delta \omega)\left(1 / 16 \pi d^{3}\right)=$ $(2 / \pi \Delta \omega) \int_{0}^{\infty} \rho^{E} \mathrm{~d} \omega$, where the last term is precisely the average electric LDOS in the large-bandwidth limit [since $H(\Delta \omega \rightarrow \infty)=1 / \pi \Delta \omega$ ]. Similarly, the $\rho^{H}$ bound only contains the $e^{-2 d \Delta \omega / c}$ factor for nonmagnetic materials, and hence the bound tends to $\left\langle\rho^{H}\right\rangle \leq 0$, in agreement with the sum rule. By construction, the bounds agree with their respective sum rules for large bandwidths.

The power-bandwidth limit of Eq. (22) applies equally well to 2D materials characterized by a spatial conductivity $\sigma(\omega)$, with the substitution $\omega \chi(\omega) \rightarrow i \delta_{S}(\mathbf{x}) \sigma(\omega)$, where $\delta_{S}(\mathbf{x})$ is a delta function on the surface of the (not necessarily planar [83]) 2D material. In doing so, the delta function transforms the volume integral in Eq. (22) to a surface integral over the incident field. We can enclose the 2D scatterer in a high-symmetry enclosure: For a 2D plane enclosure, and keeping only the highest-order terms in the near-field limit $(|\omega| d / c \ll 1)$, we find that the LDOS above the $2 \mathrm{D}$ material is bounded above by (SM [78])

$$
\begin{aligned}
\frac{\langle\rho\rangle}{\rho_{0}(|\omega|)}, \frac{\left\langle\rho^{E}\right\rangle}{\rho_{0}(|\omega|)} & \leq \frac{3}{8|k|^{4} d^{4}} f(\omega) e^{-2 d \Delta \omega / c}+\frac{1}{4|k|^{3} d^{3}} \frac{\Delta \omega}{|\omega|}, \\
\frac{\left\langle\rho^{H}\right\rangle}{\rho_{0}(|\omega|)} & \leq \frac{1}{4|k|^{2} d^{2}} f(\omega) e^{-2 d \Delta \omega / c}
\end{aligned}
$$

where for $2 \mathrm{D}$ materials the material FOM is

$$
f(\omega)=\frac{|\sigma(\omega)|^{2}}{\operatorname{Re} \sigma(\omega)} .
$$

(In SI units, there would be an additional factor of the freespace impedance $Z_{0}$ multiplying $|\sigma|^{2} / \operatorname{Re} \sigma$.) There are two distinct features that emerge for $2 \mathrm{D}$ materials: The material FOM is $|\sigma(\omega)|^{2} / \operatorname{Re} \sigma(\omega)$, and the electric- and magneticLDOS bounds have terms that scale as $1 / d^{4}$ and $1 / d^{2}$, instead of $1 / d^{3}$ and $1 / d$ in the bulk-material bounds. The different distance scaling is a natural consequence of integrating the norm of the Green's function over an area instead of a volume. Yet, there is an interesting contrast embedded within the bounds for $\langle\rho\rangle$ and $\left\langle\rho^{E}\right\rangle$ : Their first term, dominant over narrow bandwidths, scales as $1 / d^{4}$, whereas the second term, dominant over wider bandwidths and corresponding to the sum rule, scales as $1 / d^{3}$. The faster scaling with $1 / d$ of the first term suggests a scenario in which the average LDOS over a narrow bandwidth may be larger than the sum rule would seem to allow. One possibility is that the bound is "loose" and that the $1 / d^{4}$ scaling is artificial, but in multiple previous studies $[37,100]$ of singlefrequency behavior, $1 / d^{4}$ scaling has been observed in the
LDOS near 2D materials. Another possibility is that such large response is only possible over a narrow bandwidth, though the connection of broadband response to singlecomplex-frequency response would seem to suggest that large response is likely not restricted to single frequencies. Finally, perhaps the most likely possibility is that such a bound is achievable and simply requires negative (scattered) LDOS at frequencies outside the range of interest. There is no requirement that scattered LDOS be positive at all frequencies since a scatterer can suppress all modes and reduce the total LDOS to nearly zero. The idea of exploiting such suppression to achieve anomalously large response over some desired bandwidth is intriguing.

Our derivations provide general insight into optimal structures that would reach the bounds. First, as noted above, it is critical to have as much material as possible in the near-field region of the source-that material enables the polarization currents that ultimately drive the large response. Sharp tips, though potentially exhibiting strong resonances, are not ideal. Second, the convexity-based optimization provides not only the maximal bandwidthaveraged response but also the optimal fields $\psi$ that would generate such response. In all cases, those optimal fields, throughout the volume of the scatterer, are proportional to the incident fields. Hence, one would want to generate, perhaps via computational design [61,101-103], resonances with the same phase and amplitude profile as the fields emanating from the dipolar sources. Finally, through a volume-integral-equation (VIE) framework [36], one can reinterpret our bounds as upper limits that occur when the incident field couples only to a single VIE mode at the optimal resonance location. Thus, if one can completely avoid exciting other modes, then the upper bound is in fact guaranteed to be achieved.

\section{CROSS DENSITY OF STATES}

The previous section developed the theoretical framework for power-bandwidth limits in the context of LDOS. We translate that framework to other near-field optical response functions, starting with CDOS [104], which measures field correlations between two points of a structured environment. In addition to fundamental interest as a correlation function, CDOS is also the critical term in the frequency integrand for resonant near-field dipolar energy transfer, as in Förster energy transfer $[46,47,105]$, as well as for quantum entanglement and super-radiative coupling between qubits [106-110]. Whereas LDOS is given by the Green's function for identical source and measurement points, CDOS is given by the Green's function between different source and measurement points, $\mathbf{x}_{0}$ and $\mathbf{x}$, respectively [104]:

$$
\rho_{i j}\left(\mathbf{x}, \mathbf{x}_{0}, \omega\right)=\operatorname{Im}\left[\frac{1}{\pi \omega} \Gamma_{\mathrm{s}, i j}\left(\mathbf{x}, \mathbf{x}_{0}, \omega\right)\right],
$$


where $i$ and $j$ are the measurement and source polarizations, respectively, and we again use the "s" subscript to denote the scattered-field contribution (subtracting off the known free-space contribution). By analogy to Eq. (4), there is a sum-rule constant for CDOS, which we denote $\alpha_{\mathrm{CDOS}}$ (defined as $\frac{1}{2} \operatorname{Re}\left[\left.\Gamma_{\mathrm{s}, i j}\left(\mathbf{x}, \mathbf{x}_{0}\right)\right|_{\omega=0}\right]$ ); by the similarity to LDOS, one can follow a similar procedure to derive power-bandwidth limits. The key new feature is that instead of the single separation distance between the emitter and scatterer controlling the bound, now there are two relevant separation distances: the distance between the emitter and the scatterer, denoted $d_{1}$, and the distance between the scatterer and the measurement point, $d_{2}$.

To bound Eq. (26) averaged over any bandwidth, we first rewrite the Green's function in terms of the polarization currents of the scatterer(s). This leads to an overlap integral between the polarization currents induced by the field incident from the source position with a (parity-reversed) secondary field incident from the measurement position. Ideally, the polarization response is maximally aligned to both fields (SM [78]), in which case the maximal response is proportional to the square root of the energy of each

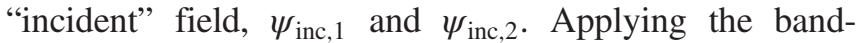
averaged bound approach modified as described above, we arrive at the following bound on average CDOS for bulk, nonmagnetic materials (SM [78]):

$$
\begin{aligned}
\left\langle\rho_{i j}\right\rangle \leq & \frac{f(\omega)}{\pi|\omega|}\left\{\left(\int_{V} \psi_{\mathrm{inc}, 1, i}^{\dagger}(\omega) \psi_{\mathrm{inc}, 1, i}(\omega) \mathrm{d} V\right)\right. \\
& \left.\times\left(\int_{V} \psi_{\mathrm{inc}, 2, j}^{\dagger}(\omega) \psi_{\mathrm{inc}, 2, j}(\omega) \mathrm{d} V\right)\right\}^{1 / 2} \\
& +2 H_{\omega_{0}, \Delta \omega}(0) \alpha_{\mathrm{CDOS}},
\end{aligned}
$$

where the complex frequency $\omega=\omega_{0}+i \Delta \omega$ encodes the center frequency and bandwidth of interest. In the nearfield regime, $(|\omega| d / c \ll 1)$, Eq. (27) further simplifies (SM [78], neglecting $\alpha_{\mathrm{CDOS}}$ for small to moderate bandwidths):

$$
\begin{aligned}
\frac{\left\langle\rho_{i j}\right\rangle}{\rho_{0}(|\omega|)}, \frac{\left\langle\rho_{i j}^{E}\right\rangle}{\rho_{0}(|\omega|)} & \leq \frac{1}{12|k|^{3} \sqrt{d_{1}^{3} d_{2}^{3}}} f(\omega) e^{-\left(d_{1}+d_{2}\right) \Delta \omega / c}, \\
\frac{\left\langle\rho_{i j}^{H}\right\rangle}{\rho_{0}(|\omega|)} & \leq \frac{1}{6|k| \sqrt{d_{1} d_{2}}} f(\omega) e^{-\left(d_{1}+d_{2}\right) \Delta \omega / c},
\end{aligned}
$$

where we separate the electric- and magnetic-source contributions to the CDOS. Now, the electric bounds depend on the source-scatterer and measurement-scatterer separation distances to the three-halves power, instead of the cubic dependence for LDOS when the source and measurement points are identical. Note that the material dependence of the bound is encoded in the same material figure of merit, $f(\omega)$, as defined in Eq. (21), suggesting the universal role it may play in determining the maximal broadband response of any material.

\section{RADIATIVE HEAT TRANSFER}

Near-field radiative heat transfer (NFRHT) can be substantially larger than far-field radiative heat transfer, via photon-tunneling evanescent-wave energy transfer, and has generated much interest for applications such as thermophotovoltaics [11,111-114]. In RHT, there are two bodies at temperatures $T_{1}$ and $T_{2}$, with minimal separation distance $d$. The net radiative heat flux between the two bodies [115] is given by $H_{1 \rightarrow 2}(\omega)=\Phi(\omega)\left[\Theta\left(\omega, T_{1}\right)-\Theta\left(\omega, T_{2}\right)\right]$, where $\Theta(\omega, T)$ denotes the mean energy of the harmonic oscillator (without the zero-point energy $\hbar \omega / 2$ ) and $\Phi$ is a temperature-independent flux rate from incoherent sources in body 1 radiating to body 2 .

Since $\Theta$ is positive for all frequencies, we can bound the difference $\Theta\left(T_{1}\right)-\Theta\left(T_{2}\right)$ by its maximum value, which is simply $\Theta\left(T_{1}\right)$ (taking $T_{1}>T_{2}$ ), i.e., ( $\Phi$ is non-negative at all frequencies for passive media):

$$
\begin{aligned}
H_{1 \rightarrow 2}= & \int_{0}^{\infty} \Phi(\omega)\left[\Theta\left(\omega, T_{1}\right)-\Theta\left(\omega, T_{2}\right)\right] d \omega \\
& \leq \int_{0}^{\infty} \Phi(\omega) \Theta(\omega, T) d \omega,
\end{aligned}
$$

where $T=T_{1}$ and the equality holds if body 2 is at absolute zero.

In order to apply contour-integration techniques (described in Sec. II C) to bound Eq. (30), one would need to extend the mean energy $\Theta$ to negative frequencies, and $\Theta$ has to decay fast enough such that the integral in Eq. (30) is finite for all real frequencies. While it is bounded and decays for large positive frequencies, it diverges when extended to negative frequencies. To avoid such asymmetry, one could add the vacuum energy $\hbar \omega / 2$ and work with $\Theta_{v}=\frac{1}{2} \hbar \omega \operatorname{coth}\left(\hbar \omega / 2 k_{B} T\right)$ [115] $\quad\left(k_{B}\right.$ denotes the Boltzmann constant), which is symmetric about the origin. However, $\Theta_{v}$ diverges linearly for large frequencies and would enforce dramatic restrictions on the flux rate $\Phi$ to fall rapidly at high frequencies. Even if convergence were not an issue, $\Theta_{v}$ contains infinitely many singularities along the imaginary axis. This would reduce a contour integral evaluation of Eq. (30) (albeit with $\Theta_{v}$ instead of $\Theta$ ) to a sum of infinitely many residues (at the "Matsubara frequencies" [116]), which is cumbersome to handle. We can avoid all of these issues (asymmetry, nonconvergence, and the Matsubara sum) in a single stroke by virtue of the following fact: For any temperature $T$, the mean-energy spectrum $\Theta(\omega, T)$ is simultaneously bounded above and closely approximated (at frequencies with non-negligible contributions) by a Lorentzian function centered at zero with bandwidth $\sqrt{2} k_{B} T / \hbar, H_{0, \sqrt{2} k_{B} T / \hbar}(\omega)$, properly scaled such that it coincides with $\Theta$ at zero frequency:

$$
\Theta(\omega, T) \leq \sqrt{2} \pi \hbar\left(\frac{k_{B} T}{\hbar}\right)^{2} H_{0, \sqrt{2} k_{B} T / \hbar}(\omega),
$$


where, for this line, $H$ again denotes the Lorentzian window function defined in Eq. (15). There is close agreement between $H_{0, \sqrt{2} k_{B} T / \hbar}(\omega)$ and $\Theta(\omega, T)$, with the total energy as measured by the integral $\int_{0}^{\infty} d \omega$ larger for the Lorentzian by only $3 \sqrt{2} / \pi \approx 1.35$ for all temperatures (cf. SM [78]). [Note that unlike the spectrum of a blackbody, whose peak wavelength is nonzero and scales inversely with temperature, the mean-energy spectrum $\Theta(\omega)$ peaks at zero frequency.]

Since the integral in Eq. (31) is the Lorentzian-averaged flux rate, it might be tempting to close the contour in the UHP to relate the integral to a sum of residues, in the spirit of Sec. II C. However, unlike LDOS, the flux rate $\Phi$ is not directly given by the real or imaginary part of a scattering amplitude. But we can transform the problem by generalized reciprocity [117], recasting the flux rate from a surface integral of fields generated by volume sources to a volume integral of fields generated by sources along a surface, revealing a surprising similarity to LDOS and ultimately leading to NFRHT bounds.

The heat flux between the two bodies is given by the power flow through a separating surface $S$ :

$$
\begin{aligned}
H_{1 \rightarrow 2}(\omega) & =\frac{1}{2} \operatorname{Re} \int_{S}(\mathbf{E} \times \overline{\mathbf{H}}) \cdot \hat{\mathbf{n}} \\
& =\frac{1}{4} \int_{S} \psi^{\dagger} \Lambda \psi,
\end{aligned}
$$

where $\Lambda=\left({ }_{\hat{\mathbf{n}} \times}{ }^{-\hat{\mathbf{n}} \times}\right)$ is a real symmetric matrix. The fields can be expressed [118] as convolutions of the system Green's function $\boldsymbol{\Gamma}\left(\mathbf{x}, \mathbf{x}^{\prime}\right)$ with the thermal sources $\phi\left(\mathbf{x}^{\prime}\right)$ : $\psi(\mathbf{x})=\int_{V} \boldsymbol{\Gamma}\left(\mathbf{x}, \mathbf{x}^{\prime}\right) \phi\left(\mathbf{x}^{\prime}\right) d \mathbf{x}^{\prime}$. The incoherence of the thermal sources can be incorporated via the fluctuationdissipation theorem in a standard substitution [115]. The key step is that we then use generalized reciprocity [117] (cf. SM [78]) to interchange the source positions in body 1 with the measurement position on the surface $S$ separating the bodies. This transforms the problem to sources over a surface in free space (or a homogeneous background) radiating into one of the bodies, and the net flux rate is an energylike quadratic form evaluated in the body, for a specific linear combination of electric and magnetic dipoles (cf. SM [78]). In the ideal scenario, the dipoles are only emitted into body 1 ; in any case, the radiation into body 1 is bounded above by the total radiation. In the near field, the total radiation is essentially exactly equal to the scattered-field LDOS, such that we can directly bound (via Cauchy-Schwarz arguments) the flux rate at any frequency by the product of the electric and magnetic LDOS (SM [78]): $\Phi(\omega) \leq 4 c \int_{S} \sqrt{\rho^{E}(\omega) \rho^{H}(\omega)}$. To bound the bandwidthaveraged flux rate $\langle\Phi\rangle$, the product of the electric and magnetic LDOS prevents direct identification of a complexanalytic quantity, but we can again use Cauchy-Schwarz for a bound in terms of the individually bandwidth-averaged
$\rho^{E}$ and $\rho^{H}$ (ideally, they would exhibit the same line shape, in which case the Cauchy-Schwarz substitution would be an equality), such that we can write (SM [78])

$$
\langle\Phi\rangle \leq 4 c \int_{S} \sqrt{\left\langle\rho^{E}\right\rangle\left\langle\rho^{H}\right\rangle} .
$$

In the case of near-field RHT, the center frequency and bandwidth are fixed by the temperature as discussed above. Using Eq. (33), we can bound NFRHT in Eq. (31):

$H_{1 \rightarrow 2} \leq 2 \sqrt{2} \pi \hbar c\left(\frac{k_{B} T}{\hbar}\right)^{2} \int_{S} \sqrt{\left\langle\rho^{E}\right\rangle\left\langle\rho^{H}\right\rangle_{0, \sqrt{2} k_{B} T / \hbar}}$.

Equation (34) is the culmination of our transformations: The product of flux and oscillator energy in the integrand of Eq. (30), which is not easily extensible to negative frequencies nor analytic over the upper-half plane, is bounded above by (and, for optimal designs, equal to) an integral over a bounding surface between the bodies of the geometric mean of the bandwidth-averaged electric and magnetic LDOS. Then, for bulk, nonmagnetic materials, we can directly insert the LDOS bounds, Eq. (23) (with the second term slightly modified to allow for a two half-spaces enclosure), into Eq. (34) to obtain a near-field bound on net radiative power transfer (with suitable generalizations for magnetic and/or 2D materials). The material figure of merit of Eq. (21) again plays a key role; in the case of near-field RHT, the temperature determines the bandwidth. Moreover, because the center frequency is zero, the material figure of merit is evaluated at the purely imaginary frequency $i \sqrt{2} k_{B} T / \hbar$; since susceptibilities are real and positive for imaginary frequencies in the UHP [79], the material FOM (now written as a function of temperature) can be greatly simplified:

$$
f(T)=\chi\left(i \sqrt{2} k_{B} T / \hbar\right) .
$$

If we denote $r_{1}$ and $r_{2}$ as the distances from a surface point to bodies 1 and 2, respectively, and $f_{1}(T)$ and $f_{2}(T)$ as the corresponding material figures of merit, then the bound for an arbitrary separating surface $S$ is

$$
\begin{aligned}
H_{1 \rightarrow 2} \leq & \frac{\rho_{0}\left(\sqrt{2} k_{B} T / \hbar\right) \hbar c^{3}}{2} \int_{S}\left\{\left(\frac{f_{1}(T) e^{-\frac{2 \sqrt{2} k_{B} T_{r_{1}}}{\hbar c}}+4.21 \ldots}{r_{1}^{3}}\right.\right. \\
& \left.+\frac{f_{2}(T) e^{-\frac{2 \sqrt{2} k_{B} T r_{2}}{\hbar c}}+4.21 \ldots}{r_{2}^{3}}\right) \\
& \left.\times\left(\frac{f_{1}(T) e^{-\frac{2 \sqrt{2} k_{B} T_{r_{1}}}{\hbar c}}}{r_{1}}+\frac{f_{2}(T) e^{-\frac{2 \sqrt{2} k_{B} T r_{2}}{\hbar c}}}{r_{2}}\right)\right\}^{1 / 2},
\end{aligned}
$$

where the constant terms proportional to 4.21 arise from the LDOS constant $\alpha_{\mathrm{LDOS}}$ for two half-spaces (SM [78]). For a 
planar bounding surface halfway between the two bodies at $d / 2$ for a minimal separation $d$, the integral in Eq. (37) can be done analytically, simplifying the bound to maximum heat transfer per unit surface area $A$ :

$$
\frac{H_{1 \rightarrow 2}}{A} \leq \frac{2}{\pi^{2} \hbar}\left(\frac{k_{B} T}{d}\right)^{2} e^{-\frac{\sqrt{2} k_{B} T d}{\hbar c}}\left[f_{1}(T)+f_{2}(T)\right],
$$

where we have dropped constant terms (which are small relative to $f_{1}$ and $f_{2}$ for all practical materials and temperatures of interest). Equation (38) represents the first general bound to near-field radiative heat transfer.

We can more easily interpret Eq. (38) by recasting the expression as a product of dimensionless enhancement factors with the far-field blackbody limit, $H_{\mathrm{BB}}=\sigma T^{4}$, where $\sigma$ is the Stefan-Boltzmann constant. Using the thermal de Broglie wavelength, $\lambda_{T}=\pi^{2 / 3} \hbar c / k_{B} T$, algebraic manipulations yield an equivalent alternative,

$\frac{H_{1 \rightarrow 2}}{A} \leq \sigma T^{4}\left\{\beta e^{-\left(\sqrt{2} k_{B} T d\right) / \hbar c}\left(\frac{\lambda_{T}}{d}\right)^{2}\left[f_{1}(T)+f_{2}(T)\right]\right\}$,

where $\beta=120 / \pi^{16 / 3} \approx 0.268$ is of order 1 . Equation (39) succinctly identifies two maximum possible enhancements beyond the blackbody limit. First, a distance-dependent enhancement $\left(\lambda_{T} / d\right)^{2}$ emerges, which accounts for the increased amplitudes of evanescent waves at shorter separations. This enhancement factor is intermediate between that appearing in the bounds for electric and magnetic LDOS $\left(1 / d^{3}\right.$ and $1 / d$, respectively), as RHT is equivalent to a combination of electric and magnetic dipolar radiation in free space. The thermal wavelength $\lambda_{T}$ (which is about $10 \mu \mathrm{m}$ at room temperature) sets the threshold for the nearfield regime, highly sensible since the blackbody radiation limit holds only when the length scales involved are greater than $\lambda_{T}$. The second enhancement factor is a materialdependent factor $f_{1}(T)+f_{2}(T)$, which accounts for material-based resonant enhancements. Per the material FOM of Eq. (35), the larger the susceptibility is at the complex frequency set by the temperature of the emitter, the larger the possible response is. The bounds of Eqs. (38), and (39) cannot be overcome by any metamaterial, metasurface, or other design approaches.

\section{OPTIMAL MATERIALS}

Embedded throughout the LDOS, CDOS, and near-field RHT bounds is a material metric $f(\omega)$, defined in Eqs. (21) and (25), that indicates the intrinsic capability of any material to exhibit large optical response over a frequency bandwidth $\Delta \omega$ around a center frequency $\omega_{0}$. This material metric enables comparison of any material-dielectric and metal, 2D and 3D, lossless and lossy-many of whose capabilities cannot be understood through single-frequency bounds or sum rules.
Sum rules, such as Eq. (5), typically have little-to-no dependence on material parameters, suggesting that different materials only alter resonant bandwidths, without impacting total optical response. Yet, this is misleading on two fronts: (1) It only applies over infinite bandwidth; over any finite bandwidth, material properties play an important role in maximal response, and (2) sum rules require susceptibilities that satisfy Kramers-Kronig relations, diminishing to zero at high frequencies. The decay-tozero requirement, though physically reasonable, means that even "dielectric" media (semiconductors, insulators, etc.) have a plasmalike response at large enough frequencies. Such response contributes to sum rules, often in a large way due to the negative susceptibility. This contribution obscures the behavior of, for example, a transparent dielectric at optical frequencies, by accounting for transitions that occur at UV and $\mathrm{x}$-ray frequencies. Thus, sum rules miss finitebandwidth effects and dramatically overestimate dielectricmaterial interactions. At the other end of the continuum, single-frequency bounds $[24,36,37,42,43]$ apply at any given frequency, but they use material loss as the intrinsic system limitation and thereby diverge for materials with vanishingly small imaginary susceptibilities (such as dielectrics). The material FOM embedded in the power-bandwidth limits does not have any of these limitations: It can account for finite bandwidths, it does not require susceptibilities that asymptotically approach zero at large frequencies, and it provides a finite bound for lossless materials for any nonzero bandwidth. Hence, $f(\omega)$ is a simple expression that enables comparison among the multitude of possible optical materials.

To gain intuition about the material FOM, we consider the small-bandwidth limit in which $\Delta \omega \ll \omega_{0}$. We delineate two types of (bulk, 3D) materials: lossy materials, with a nonzero $\operatorname{Im} \chi(\omega)$ in the small-bandwidth limit, and lossless materials, with $\operatorname{Im} \chi(\omega) \approx 0$ [and $\operatorname{Im} \chi(\omega) \ll \Delta \omega / \omega_{0}$ even in the small-bandwidth limit], as would characterize many transparent materials at optical frequencies. In the small-bandwidth limit, the material FOM is approximately given by

$$
f(\omega) \approx \begin{cases}\frac{|\chi(\omega)|^{2}}{\operatorname{Im} \chi(\omega)} & \text { lossy (e.g., metals) } \\ \frac{\omega_{0} \frac{\chi \chi(\omega)]^{2}}{\Delta \omega(\omega)+1}}{\chi(1)} & \text { lossless (dielectrics) }, \\ \frac{|\sigma(\omega)|^{2}}{\operatorname{Re} \sigma(\omega)} & \text { 2D materials }\end{cases}
$$

where we have retained the full material FOM for 2D materials since it is already simple. For high-index lossless materials, the expression would simplify even further:

$$
f(\omega) \approx \frac{\omega_{0}}{\Delta \omega} \chi(\omega) \quad \text { lossless, high-index. }
$$

For small to moderate bandwidths, a natural dichotomy emerges: Lossy materials are inherently restricted by 
material loss in $\operatorname{Im} \chi(\omega)$, whereas lossless materials are inherently limited by the relative bandwidth $\Delta \omega / \omega_{0}$. Intuitively, in simple single-mode interactions, one could interpret the figures of merit as dictating that lossy materials have maximum responses proportional to $|\chi(\omega)|$, over a bandwidth proportional to $|\chi(\omega)| / \operatorname{Im} \chi(\omega)$, whereas lossless materials have maximum responses proportional to $\chi(\omega)$, over bandwidths proportional to $\omega_{0} / \Delta \omega$. The intuitive interpretation about lossy-material bandwidths is supported by previous results in quasistatic plasmonic frameworks $[119,120]$. Of course, we make no assumption of singlemode or quasistatic behavior, and our scattering framework is valid for any number of resonances as well as more complex phenomena such as Fano interactions [121] and exceptional points $[122,123]$. And perhaps more importantly, it enables consideration of lossless and lossy media on equal footing. As discussed in the Introduction, the maximum response of lossless media has been impossible to (a)

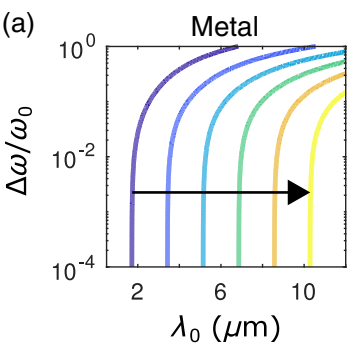

$$
f(\omega)=\frac{|\omega \chi|^{2}+|\omega \chi|\left(\omega \varepsilon_{0}\right)^{\prime \prime}}{|\omega|(\omega \varepsilon)^{\prime \prime}}
$$
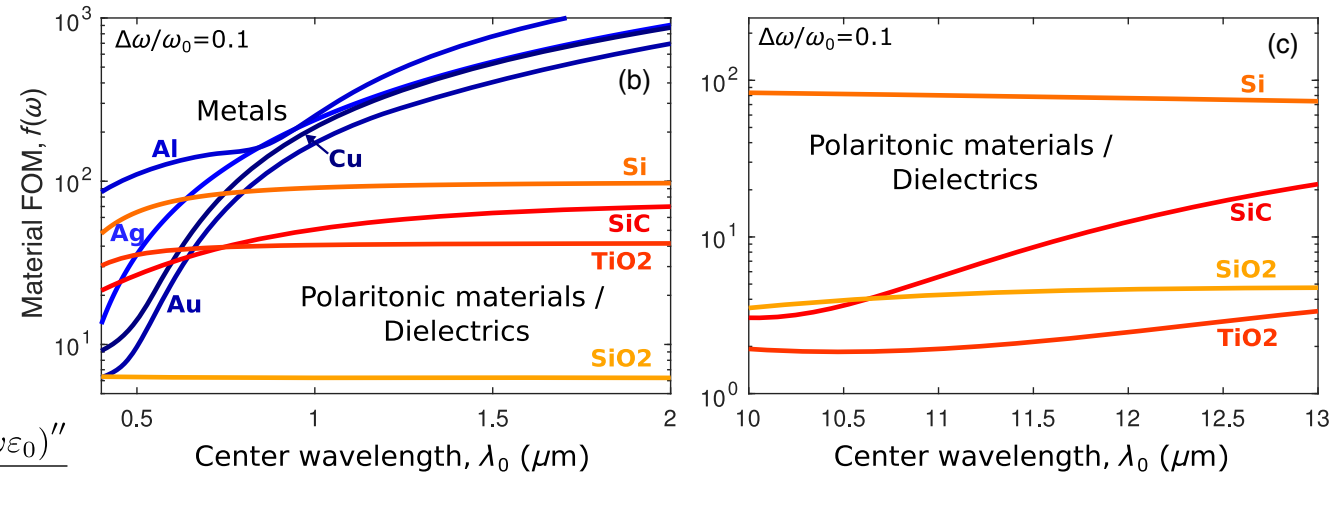

(d)
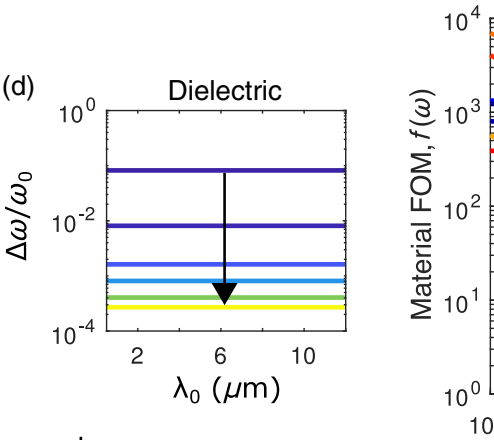

indicates direction of increasing material FOM

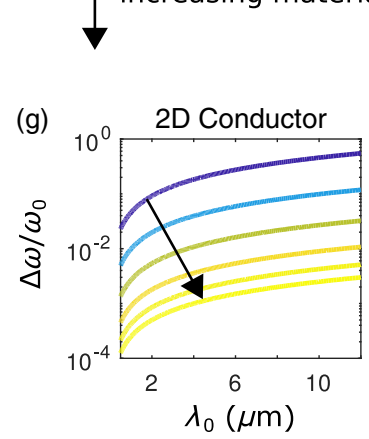

(h)

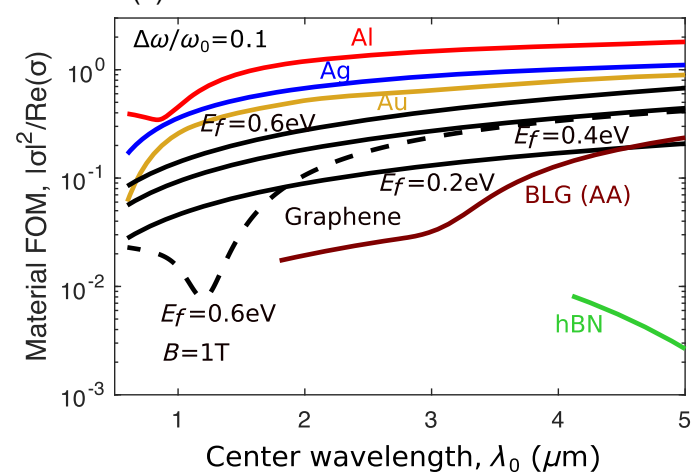

$\lambda_{0}=1.55 \mu \mathrm{m}$

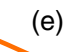

(e)
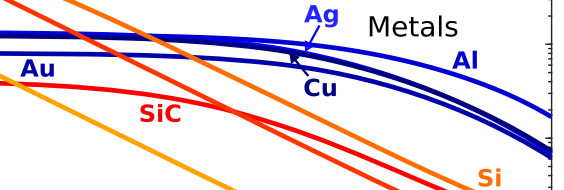

Polaritonic materials Dielectrics

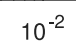

Bandwidth, $\Delta \omega / \omega_{0}$

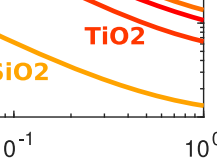

$10^{0} \quad 10^{-3}$

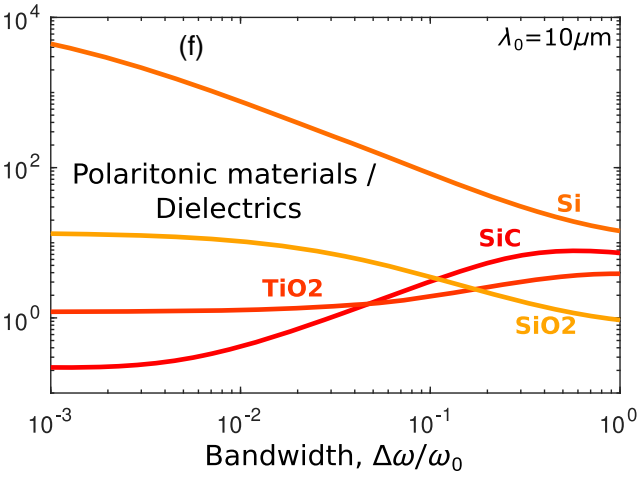

2D materials

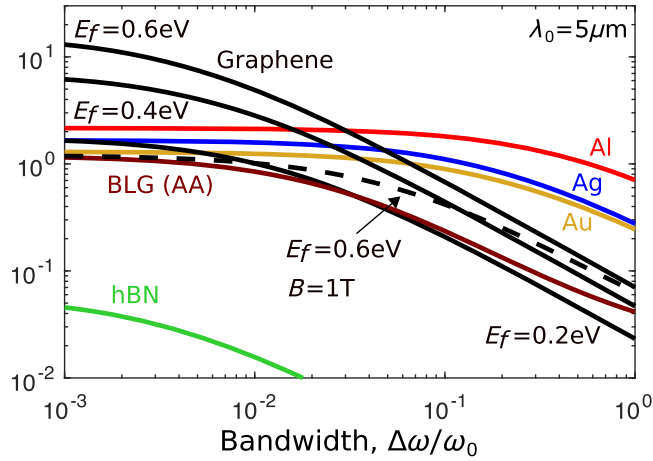

FIG. 5. (a,d,g) Isocurves of material FOM for a Drude metal (with material loss rate $\gamma=0.1 \omega_{p}$ ), a lossless dielectric (of susceptibility $\chi=9$ ), and a Drude 2D material (with $\gamma=0.01 \omega_{p}$ ). The arrows indicate increasing material FOM in each case. (b) Comparison of material FOM for various bulk metals and polaritonic materials or dielectrics, keeping the bandwidth-to-center-frequency ratio $\Delta \omega / \omega_{0}$ fixed to 0.1 . For the modestly large $10 \%$ relative bandwidth, the large susceptibilities of metals compensates for their material loss, generally resulting in greater maximum response. Part (c) compares surface-phonon-polariton-supporting materials at mid-IR wavelengths. (e,f) Comparison of material FOM for varying bandwidths relative to the center wavelengths of 1.55 and $10 \mu \mathrm{m}$. At very narrow bandwidths, dielectrics offer a greater possible response than metals. (h,i) Comparison of material FOM for 2D materials for different choices of center wavelength and $\Delta \omega / \omega_{0}$ (2D Al, $\mathrm{Ag}$, and Au properties derived from their bulk counterparts). 
accurately capture with either the sum-rule or the singlefrequency-bound approaches known today. In the complexfrequency approach, bandwidth naturally adds a form of "loss" to the system, yielding finite bounds that vary smoothly with bandwidth.

Figure 5 compares the material FOM for a large variety of materials at optical frequencies. To evaluate the material susceptibilities and conductivities at complex frequencies, we use analytic models (e.g., Lorentz-Drude oscillators) that can be continued into the complex plane, and we ensure that they are accurate over the range of bandwidths considered. On the left side of the figure, we model the material FOM for canonical material types: (a) a Drude metal, $\chi(\omega)=-\omega_{p}^{2} /\left(\omega^{2}+i \gamma \omega\right)$, for plasma frequency $\omega_{p}$ and loss rate $\gamma$, (d) a lossless, constant-susceptibility $[\chi(\omega)=9]$ material, and (g) a Drude 2D material, with conductivity $\sigma(\omega)=i \omega_{p} /(\omega+i \gamma)$. One can see that these three material types show very different characteristic dependencies of their FOM on frequency and bandwidth. The Drude-metal FOM is nearly independent of small-tomoderate bandwidths, as expected from Eq. (40)-for metals, intrinsic loss is the limiting factor. The FOM of a Drude metal increases with the center wavelength (of the frequency band of interest) $\lambda_{0}$ since the increasing wavelength increases the magnitude of the susceptibility. By contrast, a constant-permittivity "dielectric" has nearly opposite dependencies. The figure of merit is independent of center wavelength and highly dependent on the bandwidth. Because the bandwidth is the source of loss, there is a trade-off between the average response and bandwidth. Finally, 2D Drude conductors are somewhere in between.
Loss originates from both the material parameter $\gamma$ and the bandwidth, with increasing FOM towards the lower-righthand corner of Fig. 5(g): small bandwidth and large wavelength (for a large conductivity). These simplified metal, dielectric, and 2D conductor profiles capture well the key dependencies of the FOM for real materials: The plots in Figs. 5(b), 5(c), and 5(h) follow the same trends as those in Figs. 5(a), 5(d), and 5(g): Metal [81] FOM increases with wavelength, whereas dielectrics ( $\mathrm{Si}$ [124,125] and $\mathrm{SiC}$ $[126,127])$ and polaritonic materials (SiO2 [128-130] and $\mathrm{TiO} 2[131,132])$ that support surface phonon-polaritons at mid-IR frequencies [84] do not depend appreciably on wavelength. Conversely, the plots in Figs. 5(e), 5(f), 5(i) show the effects of increasing bandwidth, with metal material FOMs nearly unchanged but those of the dielectrics and polaritonic materials decreasing nearly linearly. The material FOM of 2D conductors increases with both wavelength and smaller bandwidths. We consider the 2D conductivities of graphene for various Fermi levels [82], magnetic biasing [133], and AA-type bilayer stacking (BLG) [134], hBN [135], and metals Ag, Al, and Au, with conductivities set by a combination [136] of bulk properties and interlayer atomic spacing.

An intriguing prediction that emerges from the LDOS and CDOS power-bandwidth limits is that the 2D-material bounds increase more rapidly for smaller separations $\left(\sim 1 / d^{4}\right)$ than for bulk materials $\left(\sim 1 / d^{3}\right)$, suggesting that 2D materials should overtake bulk materials as optimal, with the precise transition depending on the bound prefactors and, crucially, the relative 2D and bulk material figures of merit. In Fig. 6, we consider Drude models for

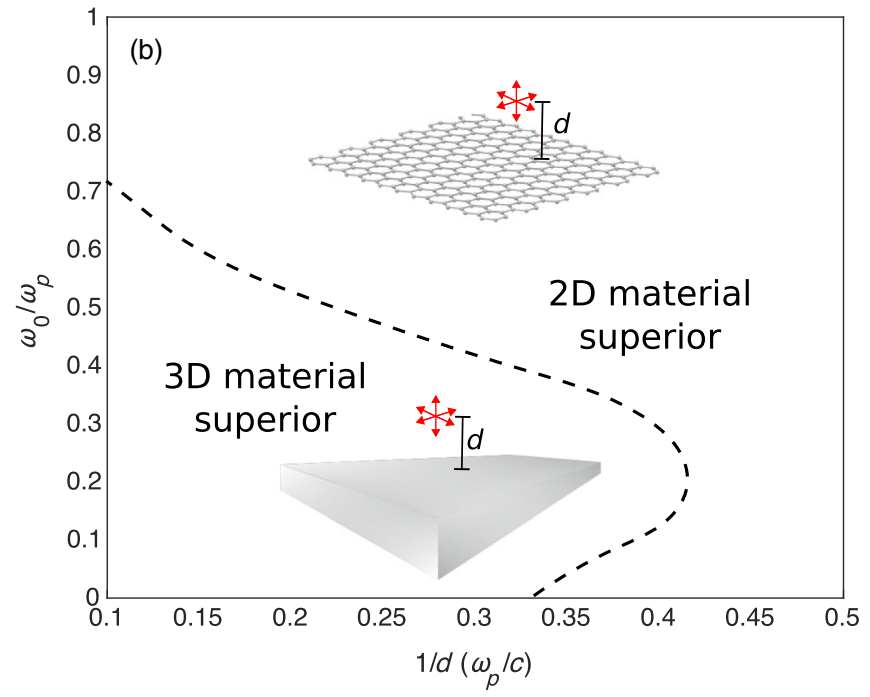

FIG. 6. Comparison of average LDOS bounds for Drude-like 3D and 2D materials with identical decay constants $\omega_{p}$ and material loss rates $\gamma=0.01 \omega_{p}$. (a) Isocurves of the average LDOS bound, which increase as both center frequency and distance decrease. (b) For a small enough emitter-scatterer distance $d, 2 \mathrm{D}$ materials are clearly superior, whereas for modest center frequencies and larger distances, $3 \mathrm{D}$ materials exhibit a larger maximum response. Such behavior arises from the fact that the bounds for $2 \mathrm{D}$ materials scale as $1 / d^{4}$, in contrast to $1 / d^{3}$ scaling for bulk materials. The dotted curve delineates the regions within which $3 \mathrm{D}$ or $2 \mathrm{D}$ materials are superior. The bandwidth-to-center-frequency ratio $\Delta \omega / \omega_{0}$ is set to 0.1 . 
both a $2 \mathrm{D}$ conductivity $\left[\sigma=i \omega_{p} /(\omega+i \gamma)\right]$ and a bulkmaterial susceptibility $\left[\chi=-\omega_{p}^{2} /\left(\omega^{2}+i \gamma \omega\right)\right]$ and plot isocontours for the material FOMs of each in panel (a). In Fig. 6(b), we trace out the region of frequency and bandwidth for which the bulk 3D material has a larger maximal response and the region for which the 2D material offers larger maximal response. This line will be different for every $2 \mathrm{D} / \mathrm{bulk}$-material pair, and it is determined by Eqs. (23) and (24) and their CDOS analogs.

The above discussion about the material FOM as a function of bandwidth is particularly relevant for LDOS and CDOS, where different source configurations lead to different center frequencies and bandwidths. For near-field RHT, as shown in Sec. IV, the center frequency is fixed (at zero), the bandwidth is uniquely determined by the temperature, and the material FOM takes a particularly simple form, $f(T)=\chi\left(i \sqrt{2} k_{B} T / \hbar\right)$. At practical temperatures of interest, the material FOM is determined by the susceptibility at an infrared frequency, except along the imaginary frequency axis instead of the real line. Dielectrics have near-constant susceptibilities over this range; surprisingly, polar dielectrics, which have resonant transitions in the infrared, corresponding to surface-phonon-polariton modes, have no resonant peaks along the imaginary axis, and exhibit dielectriclike permittivities and material FOMs. For plasmonic materials, we can use a Drude model to describe the material FOM since $k_{B} T / \hbar$ is typically an infrared frequency. For a Drude model with plasma frequency $\omega_{p}$ and loss rate $\gamma$, the material FOM is

$$
f(T)=\frac{\hbar^{2} \omega_{p}^{2}}{\sqrt{2} k_{B} T\left(\hbar \gamma+\sqrt{2} k_{B} T\right)} .
$$

Materials with large plasma frequency and small $\gamma$ are ideal for maximum heat transfer.

Figure 7 shows the material FOM for common plasmonic materials, all exhibiting a decrease with temperature. Although this may appear counterintuitive, it does not imply that maximal NFRHT decreases with temperature, as Eq. (39) has a separate $T^{4}$ multiplicative factor. Instead, such behavior reflects the fact that increasing temperature has the same effect as increasing material loss due to the larger bandwidth over which NFRHT occurs. We can understand this through two alternative vantage points: The larger bandwidth increases the imaginary part of the complex frequency at which the equivalent scattering problem occurs, and moving higher into the UHP increases loss; alternatively, in Sec. II A, we showed that LDOS quantities are subject to sum rules, and thus increasing bandwidth necessarily reduces the average response. For nondispersive dielectrics, the material FOM does not depend on temperature, and it takes on small values relative to metals (which usually have large susceptibilities at small frequencies), as shown in the inset of Fig. 7.

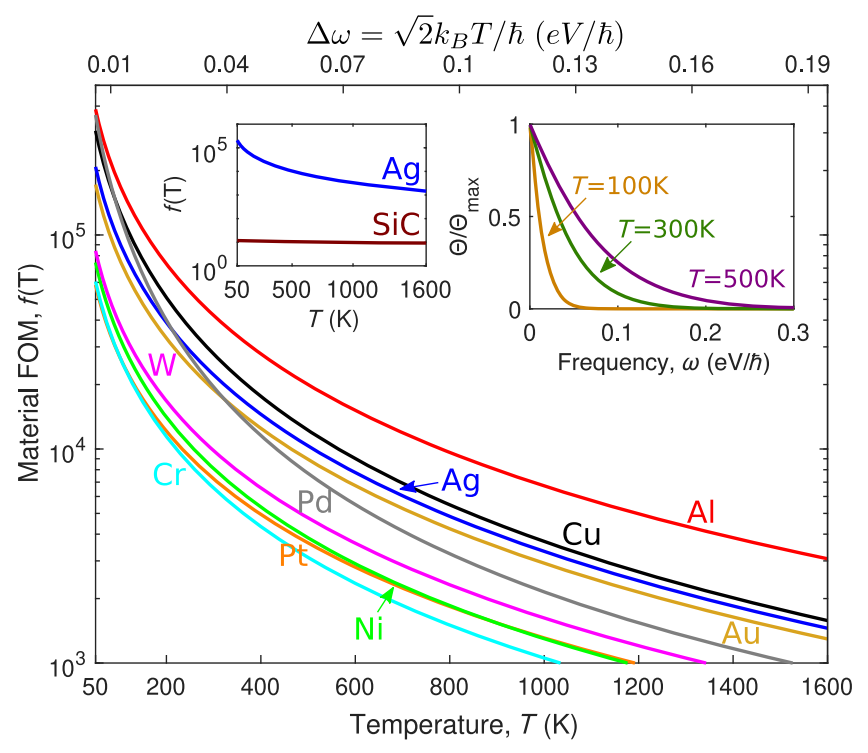

FIG. 7. Comparison of material FOM $f(T)$ in the context of NFRHT for a variety of conventional metals. The left inset shows the orders-of-magnitude difference between silver and $\mathrm{SiC}$, a polar dielectric that supports surface-phonon polaritons at infrared frequencies yet has a small material FOM due to its nonDrude-like permittivity. The right inset shows the mean energy spectrum $\Theta$ (normalized to its maximum value) at different temperatures. The decaying FOM reflects increasing loss from bandwidth, which broadens with temperature. For a practical range of temperatures as shown here, metals are superior under this metric compared to dielectrics. Generally, $f(T)$ favors materials with large plasma frequency $\omega_{p}$ and small material loss rate $\gamma$.

We can derive a particularly simple form of the NFRHT bound, Eq. (39), for plasmonic materials with $f$ given by Eq. (42). For many such materials, at practical temperatures of interest, the material loss rate $\gamma$ for many materials is much larger than $\gamma \gg k_{B} T / \hbar$. In the near field, the separation distance $d$ is much smaller than $\lambda_{T}$, such that the exponential factor in Eq. (39) is approximately 1. Assuming that both bodies consist of the same material, we can plug Eq. (42) into Eq. (39) to arrive at the following bound:

$$
\frac{H_{1 \rightarrow 2}}{A} \leq \frac{2 \sqrt{2}}{\pi^{2}} \frac{\omega_{p}^{2} k_{B} T}{\gamma d^{2}}=\sigma T^{4}\left\{\sqrt{2} \beta\left(\frac{\lambda_{T}}{d}\right)^{2} \frac{\omega_{p}}{\gamma} \frac{\omega_{p}}{k_{B} T / \hbar}\right\} .
$$

Thus, we see that there are three enhancements relative to a blackbody: the near-field distance enhancement, an enhancement from the ratio of the plasma frequency to the loss rate, and an enhancement from the ratio of the plasma frequency to an effective thermal frequency.

The material FOM extends in a natural way to anisotropic, magnetic, and even spatially inhomogeneous media, as shown in the SM [78]. Nonlocality, wherein the polarization 
field at a position $\mathbf{x}$ depends on the electromagnetic field at another $\mathbf{x}^{\prime}$, can also be incorporated for certain hydrodynamic models [137-139]. An intriguing open question is whether density functional theory models can be bounded in a similar fashion. Such bounds could motivate and clarify the search for new "quantum materials."

\section{EXTENSIONS AND SUMMARY}

We have established a framework for identifying upper bounds to near-field optical response over any frequency bandwidth of interest, with an emergent material FOM that enables quantitative comparisons of any material. We derived bounds for three optical-response functions: the LDOS, a measure of the spontaneous-emission enhancement for any electric or magnetic dipole (or atomic dipolar transition), CDOS, a field-correlation function, and radiative heat transfer, a measure of energy transfer from thermal fluctuations. The property of these response functions that is critical to our framework is the fact that they can be related to the imaginary part of a function that is analytic in the upper half of the complex-frequency plane. Here, we explore how our complex-analytic framework can be extended to other optical response functions.

There are a few near-field quantities that map closely to LDOS. First, atomic Lamb shifts due to inhomogeneous electromagnetic environments $[44,45]$ are given by frequency integrals of

$$
\operatorname{Im} \Gamma_{i j}(\mathbf{x}, \mathbf{x}, \omega),
$$

multiplied by frequency-dependent prefactors that include the atomic frequencies and position matrix elements. Hence, the sum rules and power-bandwidth limits derived here can be directly extended to the emitter-environment coupling rate in the Lamb shift. Second, Raman scattering [140] is a process in which a pump wave interacts with a molecular transition and subsequent emission that is potentially enhanced by the electromagnetic environment. It appears possible to bound this interaction above by the product of the LDOS at the separate pump and emission frequencies, in which case the framework herein can be applied for sum rules and bounds. We discuss the derivation and bounds to this process in a separate publication [141].

A more complex case is that of free-electron radiation (e.g., Smith-Purcell, Cherenkov, etc.), in which a free electron at high speed (of order $c$ ) interacts with a structured medium to generate electromagnetic radiation. The incident electromagnetic field of a free electron is proportional to a modified Bessel function. One difficulty that arises in considering sum rules and power-bandwidth limits in this case is that the modified Bessel function has a logarithmic frequency dependence at the origin, rendering it difficult to apply standard contour-integral techniques as we have done here. In two dimensions, a constant-velocity free electron emits (evanescent) plane waves, and sum rules and bounds appear to emerge in a straightforward way. The three-dimensional case may be more difficult, however.

Another complication emerges when the dipolar sources are embedded within the scatterers of interest. This is typical of the Casimir force, which is a momentum-transport quantity arising from vacuum-induced fluctuations. It appears possible to derive sum rules for such a quantity by exploiting the same generalized reciprocity [117] that we used for near-field radiative heat transfer. We will consider sum rules, powerbandwidth limits, and interesting physical consequences for Casimir physics in an upcoming publication [142].

Finally, we consider extensions of this framework to cases when the incident field is not generated by a localized dipolar or free-electron source but instead by a plane wave. At first glance, it would appear that the conventional optical theorem [33,62-64] provides a simple analytic quantity to serve as the basis for the contour-integral and energyconservation approaches developed here. Yet, the bounds derived by such an approach yield a term that grows exponentially with bandwidth [the opposite of the exponential decay seen in Eq. (22)]. Such a dependence is not physical—known sum rules [25] would contradict it—and is instead an indication that the energy constraints developed herein, based on the positive-definite quantities in Eq. (19), may not be optimal for plane-wave sources. Modified constraints may be required to develop powerbandwidth limits for plane-wave scattering.

The bounds derived here, and those suggested above, suggest a tremendous design opportunity in near-field nanophotonics. For various canonical structures, there are frequency ranges at which they come close to reaching the bounds, but there are also wide frequency ranges at which there is a sizable gap. New designs, and new approaches such as large-scale computational "inverse design" [61,101-103], offer the prospect for overcoming the gap and revealing the physical principles underlying optimal operation.

\section{ACKNOWLEDGMENTS}

We thank Yi Yang, Chia-Wei Hsu, and Thomas Christensen for helpful discussions. We thank Haejun Chung for codes to optimize material permittivity functions. H. S. and O. D. M. were supported by the Air Force Office of Scientific Research under Grant No. FA9550-171-0093. L. F. was supported by a Shanyuan Overseas grant from the Hong Kong Shanyuan Foundation at Nanjing University. S. G. J. was supported by the Army Research Office under Contracts No. W911NF-18-2-0048 and No. W911NF-13-D-0001.

[1] K. Okamoto, I. Niki, A. Shvartser, Y. Narukawa, T. Mukai, and A. Scherer, Surface-Plasmon-Enhanced Light Emitters Based on InGaN Quantum Wells, Nat. Mater. 3, 601 (2004). 
[2] D. Englund, D. Fattal, E. Waks, G. Solomon, B. Zhang, T. Nakaoka, Y. Arakawa, Y. Yamamoto, and J. Vučković, Controlling the Spontaneous Emission Rate of Single Quantum Dots in a Two-Dimensional Photonic Crystal, Phys. Rev. Lett. 95, 013904 (2005).

[3] S. Noda, M. Fujita, and T. Asano, Spontaneous-Emission Control by Photonic Crystals and Nanocavities, Nat. Photonics 1, 449 (2007).

[4] K. J. Russell, T.-L. Liu, S. Cui, and E. L. Hu, Large Spontaneous Emission Enhancement in Plasmonic Nanocavities, Nat. Photonics 6, 459 (2012).

[5] L. Novotny and B. Hecht, Principles of Nano-Optics, 2nd ed. (Cambridge University Press, Cambridge, England, 2012).

[6] G. M. Akselrod, C. Argyropoulos, T. B. Hoang, C. Ciracì, C. Fang, J. Huang, D. R. Smith, and M. H. Mikkelsen, Probing the Mechanisms of Large Purcell Enhancement in Plasmonic Nanoantennas, Nat. Photonics 8, 835 (2014).

[7] H. B. Casimir, On the Attraction between Two Perfectly Conducting Plates, Proc. Kon. Ned. Akad. Wetenschap 51, 793 (1948).

[8] E. M. Lifshitz, The Theory of Molecular Attractive Forces between Solids, Sov. Phys. JETP 2, 73 (1956).

[9] S. K. Lamoreaux, The Casimir Force: Background, Experiments, and Applications, Rep. Prog. Phys. 68, 201 (2005).

[10] J. N. Munday, F. Capasso, and V. A. Parsegian, Measured Long-Range Repulsive Casimir-Lifshitz Forces, Nature 457, 170 (2009).

[11] A. W. Rodriguez, F. Capasso, and S. G. Johnson, The Casimir Effect in Microstructured Geometries, Nat. Photonics 5, 211 (2011).

[12] M. Moskovits, Surface-Enhanced Spectroscopy, Rev. Mod. Phys. 57, 783 (1985).

[13] S. Nie and S. R. Emory, Probing Single Molecules and Single Nanoparticles by Surface-Enhanced Raman Scattering, Science 275, 1102 (1997).

[14] K. Kneipp, Y. Wang, H. Kneipp, L. T. Perelman, I. Itzkan, R. R. Dasari, and M. S. Feld, Single Molecule Detection Using Surface-Enhanced Raman Scattering (SERS), Phys. Rev. Lett. 78, 1667 (1997).

[15] R. Zhang, Y. Zhang, Z. C. Dong, S. Jiang, C. Zhang, L. G. Chen, L. Zhang, Y. Liao, J. Aizpurua, Y. Luo, J. L. Yang, and J. G. Hou, Chemical Mapping of a Single Molecule by Plasmon-Enhanced Raman Scattering, Nature 498, 82 (2013).

[16] M. Vendrell, K. K. Maiti, K. Dhaliwal, and Y.-T. Chang, Surface-Enhanced Raman Scattering in Cancer Detection and Imaging, Trends Biotechnol. 31, 249 (2013).

[17] V. V. Thacker, L. O. Herrmann, D. O. Sigle, T. Zhang, T. Liedl, J. J. Baumberg, and U.F. Keyser, DNA Origami Based Assembly of Gold Nanoparticle Dimers for SurfaceEnhanced Raman Scattering, Nat. Commun. 5, 3448 (2014).

[18] D. Polder and M. V. Hove, Theory of Radiative Heat Transfer between Closely Spaced Bodies, Phys. Rev. B 4, 3303 (1971).

[19] S. M. Rytov, Y. A. Kravtsov, and V. I. Tatarskii, Principles of Statistical Radiophysics (Springer, New York, 1988).
[20] J. B. Pendry, Radiative Exchange of Heat between Nanostructures, J. Phys. Condens. Matter 11, 6621 (1999).

[21] K. Kim, B. Song, V. Fernández-Hurtado, W. Lee, W. Jeong, L. Cui, D. Thompson, J. Feist, M. H. Reid, F. J. García-Vidal et al., Radiative Heat Transfer in the Extreme Near Field, Nature (London) 528, 387 (2015).

[22] B. Song, Y. Ganjeh, S. Sadat, D. Thompson, A. Fiorino, V. Fernández-Hurtado, J. Feist, F. J. Garcia-Vidal, J. C. Cuevas, P. Reddy et al., Enhancement of Near-Field Radiative Heat Transfer Using Polar Dielectric Thin Films, Nat. Nanotechnol. 10, 253 (2015).

[23] R. St-Gelais, L. Zhu, S. Fan, and M. Lipson, Near-Field Radiative Heat Transfer between Parallel Structures in the Deep Subwavelength Regime, Nat. Nanotechnol. 11, 515 (2016).

[24] O. D. Miller, S. G. Johnson, and A. W. Rodriguez, ShapeIndependent Limits to Near-Field Radiative Heat Transfer, Phys. Rev. Lett. 115, 204302 (2015).

[25] R. G. Gordon, Three Sum Rules for Total Optical Absorption Cross Sections, J. Chem. Phys. 38, 1724 (1963).

[26] S. D. Drell and A. C. Hearn, Exact Sum Rule for Nucleon Magnetic Moments, Phys. Rev. Lett. 16, 908 (1966).

[27] E. M. Purcell, On the Absorption and Emission of Light by Interstellar Grains, Astrophys. J. 158, 433 (1969).

[28] C. E. Detar, D. Z. Freedman, and G. Veneziano, Sum Rules for Inclusive Cross Sections, Phys. Rev. D 4, 906 (1971).

[29] I. J. R. Aitchison, Relativistic Quantum Mechanics (MacMillan, London, 1972).

[30] B. H. J. Mckellar, M. A. Box, and C. F. Bohren, Sum Rules for Optical Scattering Amplitudes, J. Opt. Soc. Am. 72, 535 (1982).

[31] C. Sohl, M. Gustafsson, and G. Kristensson, Physical Limitations on Broadband Scattering by Heterogeneous Obstacles, J. Phys. A 40, 11165 (2007).

[32] R. Carminati and J. J. Sáenz, Density of States and Extinction Mean Free Path of Waves in Random Media: Dispersion Relations and Sum Rules, Phys. Rev. Lett. 102, 093902 (2009).

[33] J. D. Jackson, Classical Electrodynamics (Wiley, New York, 2013).

[34] S. M. Barnett and R. Loudon, Sum Rule for Modified Spontaneous Emission Rates, Phys. Rev. Lett. 77, 2444 (1996).

[35] S. Sanders and A. Manjavacas, Analysis of the Limits of the Local Density of Photonic States Near Nanostructures, ACS Photonics 5, 2437 (2018).

[36] O. D. Miller, A. G. Polimeridis, M. T. H. Reid, C. W. Hsu, B. G. Delacy, J. D. Joannopoulos, M. Soljačicć, and S. G. Johnson, Fundamental Limits to Optical Response in Absorptive Systems, Opt. Express 24, 3329 (2016).

[37] O. D. Miller, O. Ilic, T. Christensen, M. T. H. Reid, H. A. Atwater, J. D. Joannopoulos, M. Soljačić́, and S. G. Johnson, Limits to the Optical Response of Graphene and Two-Dimensional Materials, Nano Lett. 17, 5408 (2017).

[38] D.-H. Kwon and D. Pozar, Optimal Characteristics of an Arbitrary Receive Antenna, IEEE Trans. Antennas Propag. 57, 3720 (2009). 
[39] Z. Ruan and S. Fan, Design of Subwavelength Superscattering Nanospheres, Appl. Phys. Lett. 98, 043101 (2011).

[40] I. Liberal, I. Ederra, R. Gonzalo, and R. W. Ziolkowski, Upper Bounds on Scattering Processes and MetamaterialInspired Structures that Reach Them, IEEE Trans. Antennas Propag. 62, 6344 (2014).

[41] J.-P. Hugonin, M. Besbes, and P. Ben-Abdallah, Fundamental Limits for Light Absorption and Scattering Induced by Cooperative Electromagnetic Interactions, Phys. Rev. B 91, 180202 (2015).

[42] Y. Yang, O. D. Miller, T. Christensen, J. D. Joannopoulos, and M. Soljačicć, Low-Loss Plasmonic Dielectric Nanoresonators, Nano Lett. 17, 3238 (2017).

[43] Y. Yang, A. Massuda, C. Roques-Carmes, S. E. Kooi, T. Christensen, S. G. Johnson, J. D. Joannopoulos, O. D. Miller, I. Kaminer, and M. Soljačić, Maximal Spontaneous Photon Emission and Energy Loss from Free Electrons, Nat. Phys. 14, 894 (2018).

[44] D. P. Fussell, R. C. Mcphedran, and C. M. D. Sterke, TwoDimensional Treatment of the Level Shift and Decay Rate in Photonic Crystals, Phys. Rev. E 72, 046605 (2005).

[45] C.-H. Chang, N. Rivera, J. D. Joannopoulos, M. Soljačicć, and I. Kaminer, Constructing "Designer Atoms" via Resonant Graphene-Induced Lamb Shifts, ACS Photonics 4, 3098 (2017).

[46] H. T. Dung, L. Knöll, and D.-G. Welsch, Intermolecular Energy Transfer in the Presence of Dispersing and Absorbing Media, Phys. Rev. A 65, 043813 (2002).

[47] J. A. Gonzaga-Galeana and J. R. Zurita-Sánchez, A Revisitation of the Förster Energy Transfer Near a Metallic Spherical Nanoparticle: (1) Efficiency Enhancement or Reduction? (2) The Control of the Förster Radius of the Unbounded Medium. (3) The Impact of the Local Density of States, J. Chem. Phys. 139, 244302 (2013).

[48] T. S. V. Zanten, A. Cambi, M. Koopman, B. Joosten, C. G. Figdor, and M. F. Garcia-Parajo, Hotspots of GPIAnchored Proteins and Integrin Nanoclusters Function as Nucleation Sites for Cell Adhesion, Proc. Natl. Acad. Sci. U.S.A. 106, 18557 (2009).

[49] L. Schermelleh, R. Heintzmann, and H. Leonhardt, A Guide to Super-Resolution Fluorescence Microscopy, J. Cell Biol. 190, 165 (2010).

[50] E. K. Lau, A. Lakhani, R. S. Tucker, and M. C. Wu, Enhanced Modulation Bandwidth of Nanocavity Light Emitting Devices, Opt. Express 17, 7790 (2009).

[51] L. Barron and A. Buckingham, Rayleigh and Raman Scattering from Optically Active Molecules, Mol. Phys. 20, 1111 (1971).

[52] D.-K. Lim, K.-S. Jeon, H. M. Kim, J.-M. Nam, and Y. D. Suh, Nanogap-Engineerable Raman-Active Nanodumbbells for Single-Molecule Detection, Nat. Mater. 9, 60 (2010).

[53] L. Spruch, Long-Range (Casimir) Interactions, Science 272, 1452 (1996).

[54] M. Bordag, U. Mohideen, and V. Mostepanenko, New Developments in the Casimir Effect, Phys. Rep. 353, 1 (2001).

[55] P. Ball, Fundamental Physics: Feel the Force, Nature (London) 447, 772 (2007).
[56] A. W. Rodriguez, F. Capasso, and S. G. Johnson, The Casimir Effect in Microstructured Geometries, Nat. Photonics 5, 211 (2011).

[57] S. G. Johnson, Numerical Methods for Computing Casimir Interactions, in Casimir Physics, Vol. 834, edited by D. Dalvit, P. Milonni, D. Roberts, and F. da Rosa (Springer, New York, 2011).

[58] M. T. H. Reid, J. White, and S. G. Johnson, Fluctuating Surface Currents: An Algorithm for Efficient Prediction of Casimir Interactions among Arbitrary Materials in Arbitrary Geometries, Phys. Rev. A 88, 022514 (2013).

[59] Z.-J. Yang, T. J. Antosiewicz, R. Verre, S. P. Apell, F. J. G. D. Abajo, and M. Käll, Ultimate Limit of Light Extinction by Nanophotonic Structures, Nano Lett. 15, 7633 (2015).

[60] R. E. Hamam, A. Karalis, J. D. Joannopoulos, and M. Soljačicć, Coupled-Mode Theory for General Free-Space Resonant Scattering of Waves, Phys. Rev. A 75, 053801 (2007).

[61] X. Liang and S. G. Johnson, Formulation for Scalable Optimization of Microcavities via the Frequency-Averaged Local Density of States, Opt. Express 21, 30812 (2013).

[62] R. G. Newton, Optical Theorem and Beyond, Am. J. Phys. 44, 639 (1976).

[63] C. F. Bohren, E. E. Clothiaux, and D. R. Huffman, Absorption and Scattering of Light by Small Particles (Wiley-VCH, New York, 1983).

[64] D. R. Lytle, P. S. Carney, J. C. Schotland, and E. Wolf, Generalized Optical Theorem for Reflection, Transmission, and Extinction of Power for Electromagnetic Fields, Phys. Rev. E 71, 056610 (2005).

[65] H. M. Nussenzveig, Causality and Dispersion Relations (Academic, New York, 1972).

[66] O. J. F. Martin and N. B. Piller, Electromagnetic Scattering in Polarizable Backgrounds, Phys. Rev. E 58, 3909 (1998).

[67] G. D’Aguanno, N. Mattiucci, M. Centini, M. Scalora, and M. J. Bloemer, Electromagnetic Density of Modes for a Finite-Size Three-Dimensional Structure, Phys. Rev. E 69, 057601 (2004).

[68] K. Joulain, R. Carminati, J.-P. Mulet, and J.-J. Greffet, Definition and Measurement of the Local Density of Electromagnetic States Close to an Interface, Phys. Rev. B 68, 245405 (2003).

[69] S. Scheel, Sum Rule for Local Densities of States in Absorbing Dielectrics, Phys. Rev. A 78, 013841 (2008).

[70] A. Taflove, A. Oskooi, and S. G. Johnson, Advances in FDTD Computational Electrodynamics: Photonics and Nanotechnology (Artech House, Boston, 2013), Chap. 4, pp. 65-100.

[71] E. M. Purcell, H. C. Torrey, and R. V. Pound, Resonance Absorption by Nuclear Magnetic Moments in a Solid, Phys. Rev. 69, 37 (1946).

[72] K. H. Drexhage, IV Interaction of Light with Monomolecular Dye Layers, Prog. Opt. 12, 163 (1974).

[73] E. Yablonovitch, Inhibited Spontaneous Emission in SolidState Physics and Electronics, Phys. Rev. Lett. 58, 2059 (1987).

[74] M. S. Eggleston and M. C. Wu, Efficient Coupling of an Antenna-Enhanced NanoLED into an Integrated InP Waveguide, Nano Lett. 15, 3329 (2015). 
[75] D. M. Callahan, J. N. Munday, and H. A. Atwater, Solar Cell Light Trapping beyond the Ray Optic Limit, Nano Lett. 12, 214 (2012).

[76] F. Wijnands, J. B. Pendry, F. J. Garcia-Vidal, P. M. Bell, P. J. Roberts, and L. M. Moreno, Green's Functions for Maxwell's Equations: Application to Spontaneous Emission, Opt. Quantum Electron. 29, 199 (1997).

[77] Y. Xu, R. K. Lee, and A. Yariv, Quantum Analysis and the Classical Analysis of Spontaneous Emission in a Microcavity, Phys. Rev. A 61, 033807 (2000).

[78] See Supplemental Material at http://link.aps.org/ supplemental/10.1103/PhysRevX.9.011043 for more details on our sum rule, monotonicity theorem, powerbandwidth limits, and other relevant discussions on LDOS, near-field RHT, and CDOS.

[79] L. D. Landau and E. M. Lifshitz, Electrodynamics of Continuous Media (Pergamon, Amsterdam, 1984).

[80] J. R. Schulenberger and C.H. Wilcox, The Limiting Absorption Principle and Spectral Theory for Steady-State Wave Propagation in Inhomogeneous Anisotropic Media, Arch. Ration. Mech. Anal. 41, 46 (1971).

[81] E. D. Palik, Handbook of Optical Constants of Solids (Academic Press, New York, 2003).

[82] M. Jablan, H. Buljan, and M. Soljačicć, Plasmonics in Graphene at Infrared Frequencies, Phys. Rev. B 80, 245435 (2009).

[83] T. Christensen, A.-P. Jauho, M. Wubs, and N. A. Mortensen, Localized Plasmons in Graphene-Coated Nanospheres, Phys. Rev. B 91, 165407 (2015).

[84] S. A. Maier, Plasmonics: Fundamentals and Applications (Springer, New York, 2007).

[85] D. S. Jones, Scattering by Inhomogeneous Dielectric Particles, Q. J. Mech. Appl. Math. 38, 135 (1985).

[86] D. Sjöberg, Variational Principles for the Static Electric and Magnetic Polarizabilities of Anisotropic Media with Perfect Electric Conductor Inclusions, J. Phys. A 42, 335403 (2009).

[87] S. G. Johnson, M. Ibanescu, M. A. Skorobogatiy, O. Weisberg, J. D. Joannopoulos, and Y. Fink, Perturbation Theory for Maxwell's Equations with Shifting Material Boundaries, Phys. Rev. E 65, 066611 (2002).

[88] O. D. Miller, Photonic Design: From Fundamental Solar Cell Physics to Computational Inverse Design, Ph.D. thesis, University of California, Berkeley, 2012.

[89] R. D. Grober, R. J. Schoelkopf, and D. E. Prober, Optical Antenna: Towards a Unity Efficiency Near-Field Optical Probe, Appl. Phys. Lett. 70, 1354 (1997).

[90] M. T. H. Reid and S. G. Johnson, Efficient Computation of Power, Force, and Torque in BEM Scattering Calculations, IEEE Trans. Antennas Propag. 63, 3588 (2015).

[91] M. T. H. Reid, Scuff-EM: Free, Open-Source BoundaryElement Software, http://homerreid.com/scuff-EM.

[92] R. F. Harrington, Field Computation by Moment Methods (IEEE, New York, 2000).

[93] D. M. Callahan, J. N. Munday, and H. A. Atwater, Solar Cell Light Trapping beyond the Ray Optic Limit, Nano Lett. 12, 214 (2012).
[94] H. Hashemi, C.-W. Qiu, A. P. Mccauley, J. D. Joannopoulos, and S. G. Johnson, Diameter-Bandwidth Product Limitation of Isolated-Object Cloaking, Phys. Rev. A 86, 013804 (2012).

[95] E. J. Rothwell and M. J. Cloud, Electromagnetics (CRC Press, Boca Raton, 2009).

[96] A. Welters, Y. Avniel, and S. G. Johnson, Speed-of-Light Limitations in Passive Linear Media, Phys. Rev. A 90, 023847 (2014).

[97] J. I. Gersten, The Effect of Surface Roughness on Surface Enhanced Raman Scattering, J. Chem. Phys. 72, 5779 (1980).

[98] T. H. Wood, Role of Atomic-Scale Roughness in SurfaceEnhanced Raman Scattering, Phys. Rev. B 24, 2289 (1981).

[99] O. D. Miller, C. W. Hsu, M. T. H. Reid, W. Qiu, B. G. DeLacy, J. D. Joannopoulos, M. Soljačić, and S. G. Johnson, Fundamental Limits to Extinction by Metallic Nanoparticles, Phys. Rev. Lett. 112, 123903 (2014).

[100] F. H. L. Koppens, D. E. Chang, and F. J. G. D. Abajo, Graphene Plasmonics: A Platform for Strong Light-Matter Interactions, Nano Lett. 11, 3370 (2011).

[101] J. S. Jensen and O. Sigmund, Topology Optimization for Nano-Photonics, Laser Photonics Rev. 5, 308 (2011).

[102] C. M. Lalau-Keraly, S. Bhargava, O. D. Miller, and E. Yablonovitch, Adjoint Shape Optimization Applied to Electromagnetic Design, Opt. Express 21, 21693 (2013).

[103] A. Y. Piggott, J. Lu, K. G. Lagoudakis, J. Petykiewicz, T. M. Babinec, and J. Vučković, Inverse Design and Demonstration of a Compact and Broadband On-Chip Wavelength Demultiplexer, Nat. Photonics 9, 374 (2015).

[104] A. Cazé, R. Pierrat, and R. Carminati, Spatial Coherence in Complex Photonic and Plasmonic Systems, Phys. Rev. Lett. 110, 063903 (2013).

[105] D. Martín-Cano, L. Martín-Moreno, F. J. García-Vidal, and E. Moreno, Resonance Energy Transfer and Superradiance Mediated by Plasmonic Nanowaveguides, Nano Lett. 10, 3129 (2010).

[106] J. Kästel and M. Fleischhauer, Suppression of Spontaneous Emission and Superradiance over Macroscopic Distances in Media with Negative Refraction, Phys. Rev. A 71, 011804 (2005).

[107] J. Kästel and M. Fleischhauer, Quantum Electrodynamics in Media with Negative Refraction, Laser Phys. 15, 135 (2005).

[108] D. Dzsotjan, A. S. Sørensen, and M. Fleischhauer, Quantum Emitters Coupled to Surface Plasmons of a Nanowire: A Green's Function Approach, Phys. Rev. B 82, 075427 (2010).

[109] D. Martín-Cano, A. González-Tudela, L. Martín-Moreno, F. J. García-Vidal, C. Tejedor, and E. Moreno, DissipationDriven Generation of Two-Qubit Entanglement Mediated by Plasmonic Waveguides, Phys. Rev. B 84, 235306 (2011).

[110] A. Gonzalez-Tudela, D. Martin-Cano, E. Moreno, L. Martin-Moreno, C. Tejedor, and F. J. Garcia-Vidal, Entanglement of Two Qubits Mediated by One-Dimensional Plasmonic Waveguides, Phys. Rev. Lett. 106, 020501 (2011). 
[111] M. D. Whale and E. G. Cravalho, Modeling and Performance of Microscale Thermophotovoltaic Energy Conversion Devices, IEEE Trans. Energy Convers. 17, 130 (2002).

[112] M. Laroche, R. Carminati, and J.-J. Greffet, Near-Field Thermophotovoltaic Energy Conversion, J. Appl. Phys. 100, 063704 (2006).

[113] S. Basu, Z. M. Zhang, and C. J. Fu, Review of Near-Field Thermal Radiation and Its Application to Energy Conversion, Int. J. Energy Res. 33, 1203 (2009).

[114] P. Bermel, M. Ghebrebrhan, W. Chan, Y.X. Yeng, M. Araghchini, R. Hamam, C. H. Marton, K. F. Jensen, M. Soljačić, J. D. Joannopoulos, S. G. Johnson, and I. Celanovic, Design and Global Optimization of HighEfficiency Thermophotovoltaic Systems, Opt. Express 18, A314 (2010).

[115] K. Joulain, J.-P. Mulet, F. Marquier, R. Carminati, and J.-J. Greffet, Surface Electromagnetic Waves Thermally Excited: Radiative Heat Transfer, Coherence Properties and Casimir Forces Revisited in the Near Field, Surf. Sci. Rep. 57, 59 (2005).

[116] H. Bruus and K. Flensberg, Many-Body Quantum Theory in Condensed Matter Physics: An Introduction (Oxford University, New York, 2004).

[117] J. A. Kong, Theory of Electromagnetic Waves (WileyInterscience, New York, 1975), Vol. 1.

[118] W. C. Chew, Waves and Fields in Inhomogeneous Media (IEEE, New York, 1995).

[119] F. Wang and Y. R. Shen, General Properties of Local Plasmons in Metal Nanostructures, Phys. Rev. Lett. 97, 206806 (2006).

[120] A. Raman, W. Shin, and S. Fan, Upper Bound on the Modal Material Loss Rate in Plasmonic and Metamaterial Systems, Phys. Rev. Lett. 110, 183901 (2013).

[121] U. Fano, Effects of Configuration Interaction on Intensities and Phase Shifts, Phys. Rev. 124, 1866 (1961).

[122] T. Kato, Perturbation Theory for Linear Operators (Springer, New York, 1995).

[123] W. D. Heiss, Exceptional Points of Non-Hermitian Operators, J. Phys. A 37, 2455 (2004).

[124] D. Chandler-Horowitz and P. M. Amirtharaj, HighAccuracy, Midinfrared Refractive Index Values of Silicon, J. Appl. Phys. 97, 123526 (2005).

[125] M. A. Green, Self-Consistent Optical Parameters of Intrinsic Silicon at 300K Including Temperature Coefficients, Sol. Energy Mater. Sol. Cells 92, 1305 (2008).

[126] M. Francoeur, M. P. Mengüç, and R. Vaillon, Spectral Tuning of Near-Field Radiative Heat Flux between Two Thin Silicon Carbide Films, J. Phys. D 43, 075501 (2010).
[127] J. I. Larruquert, A. P. Pérez-Marín, S. García-Cortés, L. R.-D. Marcos, J. A. Aznárez, and J. A. Méndez, SelfConsistent Optical Constants of SiC Thin Films, J. Opt. Soc. Am. A 28, 2340 (2011).

[128] I. H. Malitson, Interspecimen Comparison of the Refractive Index of Fused Silica, J. Opt. Soc. Am. 55, 1205 (1965).

[129] S. Popova, T. Tolstykh, and V. Vorobev, Optical Characteristics of Amorphous Quartz in the 1400-200 cm-1 Region, Opt. Spectrosc. 33, 444 (1972).

[130] R. Kitamura, L. Pilon, and M. Jonasz, Optical Constants of Silica Glass from Extreme Ultraviolet to Far Infrared at Near Room Temperature, Appl. Opt. 46, 8118 (2007).

[131] J. R. Devore, Refractive Indices of Rutile and Sphalerite, J. Opt. Soc. Am. A 41, 416 (1951).

[132] T. Siefke, S. Kroker, K. Pfeiffer, O. Puffky, K. Dietrich, D. Franta, I. Ohlídal, A. Szeghalmi, E.-B. Kley, A. Tünnermann, Materials Pushing the Application Limits of Wire Grid Polarizers Further into the Deep Ultraviolet Spectral Range, Adv. Opt. Mater. 4, 1780 (2016).

[133] G. W. Hanson, Dyadic Green's Functions for an Anisotropic, Non-local Model of Biased Graphene, IEEE Trans. Antennas Propag. 56, 747 (2008).

[134] W. Wang, S. Xiao, and N. A. Mortensen, Localized Plasmons in Bilayer Graphene Nanodisks, Phys. Rev. B 93, 165407 (2016).

[135] V. W. Brar, M. S. Jang, M. Sherrott, S. Kim, J. J. Lopez, L. B. Kim, M. Choi, and H. Atwater, Hybrid SurfacePhonon-Plasmon Polariton Modes in Graphenel Monolayer h-BN Heterostructures, Nano Lett. 14, 3876 (2014).

[136] F. J. G. D. Abajo and A. Manjavacas, Plasmonics in Atomically Thin Materials, Faraday Discuss. 178, 87 (2015).

[137] C. Ciracì, R. T. Hill, J. J. Mock, Y. Urzhumov, A. I. Fernández-Domínguez, S. A. Maier, J. B. Pendry, A. Chilkoti, and D. R. Smith, Probing the Ultimate Limits of Plasmonic Enhancement, Science 337, 1072 (2012).

[138] N. A. Mortensen, S. Raza, M. Wubs, T. Søndergaard, and S. I. Bozhevolnyi, A Generalized Non-local Optical Response Theory for Plasmonic Nanostructures, Nat. Commun. 5, 3809 (2014).

[139] S. Raza, S. I. Bozhevolnyi, M. Wubs, and N. A. Mortensen, Nonlocal Optical Response in Metallic Nanostructures, J. Phys. Condens. Matter 27, 183204 (2015).

[140] R. W. Boyd, Nonlinear Optics (Academic Press, New York, 2003).

[141] J. Michon et al. (unpublished).

[142] H. Shim et al. (unpublished). 